JOURNAL OF THE

AMERICAN MATHEMATICAL SOCIETY

Volume 12, Number 3, Pages 703-728

S 0894-0347(99)00302-1

Article electronically published on April 23, 1999

\title{
SEPARATION OF SEMIALGEBRAIC SETS
}

\author{
F. ACQUISTAPACE, C. ANDRADAS, AND F. BROGLIA
}

\section{INTRODUCTION}

In this paper we consider the following question: Let $M \subset \mathbb{R}^{n}$ be an irreducible algebraic variety, and $A, B \subset M$ two disjoint semialgebraic sets. When does there exist a regular function $f$ on $M$ which separates $A$ and $B$, i.e. such that $f(A)>0$ and $f(B)<0$ ? Here by an algebraic variety we mean the zero set of a finite number of polynomials and we remember that a subset $S \subset M$ is called semialgebraic if it is of the form:

$$
S=\bigcup_{i=1}^{p}\left\{x \in M \mid f_{i 1}(x)>0, \ldots, f_{i r_{i}}(x)>0, g_{i}(x)=0\right\},
$$

where $g_{i}, f_{i j} \in \mathbb{R}\left[X_{1}, \ldots, X_{n}\right]$.

We will show that the separation problem is decidable, in the sense that there exists a decision procedure (theoretical algorithm) which takes $M, A$ and $B$ as input and answers YES or NO according to whether $A$ and $B$ can be separated.

This question has attracted quite a lot of attention during the last two decades, and several partial results on separation of semialgebraic sets are already in the literature: in [Mo], Mostowski shows that if $A$ and $B$ are closed disjoint and one of them is compact, then the separation is possible, and Ruiz [Rz] shows that the same happens if one of them is one dimensional. In a different direction, in [BoEf] it is shown that separation is always possible if we allow as separating functions not only regular functions but also iterations of square roots of nonnegative polynomials, and in $[\mathrm{BrSt}]$ some study is done relating the number of necessary such square roots and the complexity of $A$ and $B$.

It is very easy to find examples of semialgebraic sets which cannot be separated by polynomials. See, for instance, Figure 1. Although they seem different these examples are quite similar. Indeed, in a neighbourhood of the point at infinity of the one-point compactification of $\mathbb{R}^{2}$, the shapes of sets $A$ and $B$ in the picture on the right in Figure 1 look like the left-hand side of Figure 2. Now, if we blow up this point, we get the configuration of the right hand side, which obeys the same pattern of the first example above: there is an irreducible component of the boundary which has the property that any possible function separating $A$ and $B$

Received by the editors February 3, 1997 and, in revised form, August 31, 1998.

1991 Mathematics Subject Classification. Primary 14P10.

This work was partially supported by EC contract CHRX-CT94-0506.

The first and third authors are members of GNSAGA of CNR, and were partially supported by MURST.

The second author was partially supported by DGICYT PB95-0354 and the Fundación del Amo, UCM. 

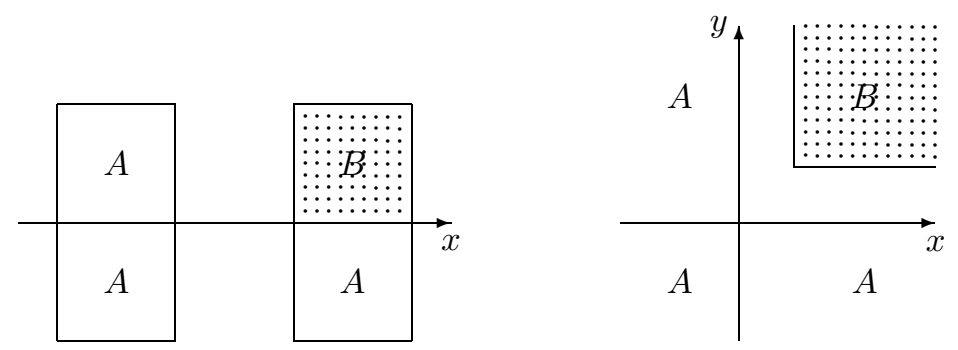

FiguRE 1
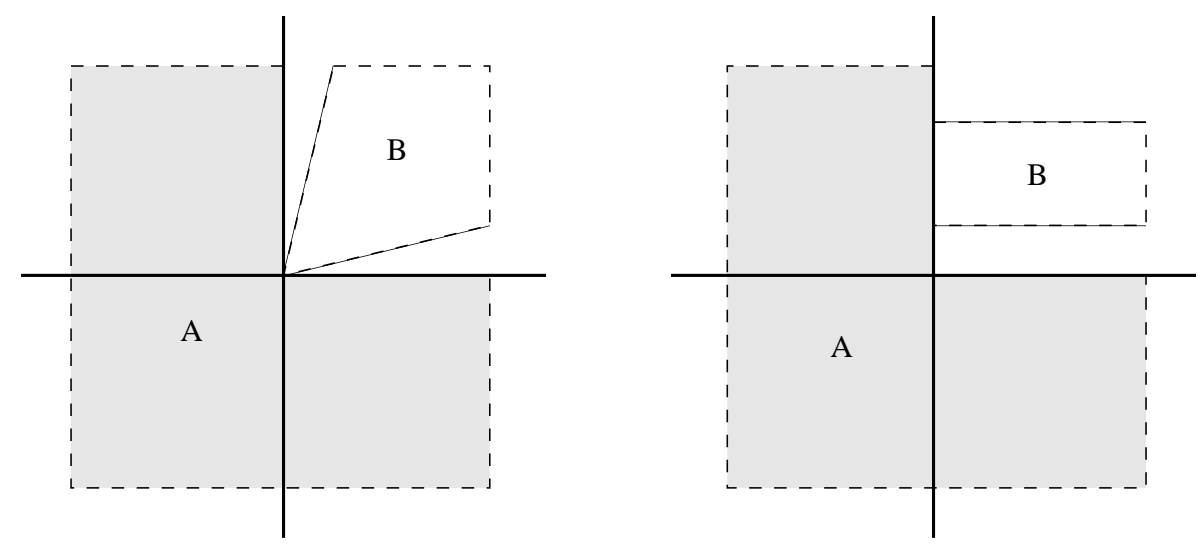

FiguRE 2

must vanish on it with multiplicity odd on one hand and even on the other, so that the separation is actually impossible.

This suggests that the relevant information for separation is at the boundary of $A$ and $B$ (including the points at infinity), although, as in the examples above, this information may be hidden and appears only after some blowing-ups, in a model where $A$ and $B$ are "fully" displayed. In fact, the main body of the paper is devoted to characterizing the generic separation of $A$ and $B$ in terms of their boundary (cf. Theorems 3.8 and 4.5 below). Here, by generic separation we mean up to an algebraic set of codimension 1 . Thus we divide our analysis of the separation procedure into two steps:

I: Study of Generic Separation.

II: Analysis of Generic Separation versus Separation.

Generic separation is a birational problem, that is, it depends only on the field $K$ of rational functions on $M$ and in its study we use the space of orderings $\Sigma_{K}$ of $K$. We remember that to any semialgebraic set $S \subset M$ we may associate the constructible set $\widetilde{S} \subset \Sigma_{K}$ defined by the same formula and that two semialgebraic sets have the same tilde image if and only if they are generically equal, i.e. they are equal up to a subset of codimension at least one [BCR, Proposition 7.6.3]. Thus the study of constructible subsets of $\Sigma_{M}$ translates into generic properties of semialgebraic sets and in particular we have that $A$ and $B$ are generically separable if and only if $\widetilde{A}$ and $\widetilde{B}$ are separable in $\Sigma_{M}$. Now, Bröcker's theorem (cf. [Br3], 
[AnBrRz, Theorem IV.7.12]) states that $\widetilde{A}$ and $\widetilde{B}$ cannot be separated if and only if there exists a finite subspace $X$ of $\Sigma_{K}$, in which $\widetilde{A}$ and $\widetilde{B}$ cannot be separated. In this way the problem is reduced, at least theoretically, to an infinite number of combinatorial ones, but still far away from being decidable.

Our approach is as follows: first we isolate a "nice" class of spaces of orderings which suffices to test the separation of $A$ and $B$. These are the Geometric Spaces of Orderings (GSO for short), which are introduced in Section 1. Roughly speaking they are spaces of orderings attached to discrete valuation rings and therefore they behave specially well with respect to the geometry of the variety, so that they easily translate the "abstract" results into geometric statements. GSO are the natural generalization of the notion of algebroid fans considered in [AnRz2]. In Section 2 we prove one of the key results of the paper: the family of GSO is dense in the one of all spaces of orderings (Theorem 2.1). In particular this implies that GSO are enough to check the separation of $\widetilde{A}$ and $\widetilde{B}$ (Theorem 2.4). In Section 3 we use GSO to show that $A$ and $B$ are generically separated if and only if, for any model of $M$, they are separated in a sort of "infinitesimal" neighbourhood of their walls (the codimension 1 components of their boundaries) making precise the idea that obstacles to separation appear in the boundary. In particular in Theorem 3.8 we show the universal obstruction to separation: $A$ and $B$ are not generically separable if and only if in some model of $M$ there is a component of the boundary of $A$ and $B$ which is simultaneously odd and even; cf. Definition 3.5.

In Section 4 we show first that generic separation can be tested in a fixed model of $M$, provided that it is nonsingular and the walls of $A$ and $B$ are at normal crossings. Then, under these assumptions we develop a geometric criterion (Theorem 4.5) which reduces the question of the generic separation of $A$ and $B$ in a neighbourhood of a wall $W$ to the separation of their shadows and counter-shadows in $W$. In this way we lower the dimension by one and by induction we get a decision procedure for the generic separation (Theorem 5.1). This geometric criterion was proved for dimension 3 in $[\mathrm{AcBgFo}]$ by a different method.

Finally in Section 6 we detect the obstructions for two generically separable semialgebraic sets $A$ and $B$ to be separable, and show that they can be recursively tested, yielding the announced result on the decidibility of the separation of semialgebraic sets of an affine variety over the real numbers (Theorem 6.6).

We want to thank A. Prestel who explained the notion of recursive enumerability and the Model Theory aspects of the decision procedure during his visit to the University of Pisa. Also, this paper was completed while the second author enjoyed a stay at Stanford University partially supported by the "Fundación del Amo" of the Universidad Complutense of Madrid. He sincerely wants to thank both institutions for their help and warm atmosphere.

\section{NotATION AND PRELIMINARIES}

The abstract theory of spaces of orderings was developed by Marshall in the series of papers [Mr1, Mr2, Mr3, Mr4]. For a self-contained new presentation see $[\mathrm{AnBrRz}]$ and $[\mathrm{Mr} 5]$. Here we just fix notations and definitions.

Let $K$ be a field, and consider its space of orderings $\Sigma_{K}=\operatorname{Spec}_{r}(K)$. Any element $\sigma \in \Sigma_{K}$ can be seen either as the cone in $K$ of all functions $f$ positive in $\sigma$, or as a signature $\sigma: K^{*} / \Sigma K^{2} \rightarrow \mathbb{Z}_{2}=\{-1,1\}$ which maps the class of the element $f \in K$ to 1 or -1 according to whether $f$ is positive or negative in $\sigma$. Thus, $\Sigma_{K}$ 
is a subset of the character group of $G_{K}=K^{*} / \Sigma K^{2}$, and the pair $\left(\Sigma_{K}, G_{K}\right)$, is an abstract Space of Orderings in the sense of Marshall. To keep a geometrical meaning in the notation we will write $f(\sigma)>0$ instead of $\sigma(f)=1$ and $f(\sigma)<0$ instead of $\sigma(f)=-1$.

A constructible subset of $\Sigma_{K}$ is a set of the form

$$
C=\bigcup_{i=1}^{p}\left\{\sigma \in \Sigma_{K} \mid f_{i 1}(\sigma)>0, \ldots, f_{i r_{i}}(\sigma)>0\right\}
$$

where $f_{i j} \in K$.

Given $E \subset K^{*}$ and $Y \subset \Sigma_{K}$, one defines

$$
\begin{aligned}
& E^{\perp}=\left\{\sigma \in \Sigma_{K} \mid f(\sigma)>0 \text { for all } f \in E\right\}, \\
& Y^{\perp}=\left\{f \in K^{*} \mid f(\sigma)>0 \text { for all } \sigma \in Y\right\}=\bigcap_{\sigma \in Y} \sigma .
\end{aligned}
$$

A subset $Y \subset \Sigma_{K}$ is called a subspace if it satisfies $Y^{\perp \perp}=Y$. In this case the pair $\left(Y, G_{K} / Y^{\perp}\right)$ is also a subspace of orderings in the sense of Marshall.

The structural theorem of finite spaces of orderings ([AnBrRz, Theorem IV.5.1], [Mr5, Theorem 4.2.2]) asserts that any finite subspace can be built in a unique way (up to isomorphism) by a finite sequence of sums and extensions, starting from a finite number of atomic spaces, i.e. with a single element. Thus, any finite space of orderings is represented, up to isomorphism, by a structure tree where the bottom points represent atomic spaces. Structure trees of finite spaces of orderings allow us to work by induction along the tree, either to define properties or to prove them.

We say that the valuation ring $V$ is compatible with a subspace of orderings $X \subset \Sigma$ if $V$ is compatible with every ordering $\sigma \in X$, that is, $V$ is convex with respect to every $\sigma$; cf. [BCR, Chapter 10]. The specializations of the orderings of $X$ form a subspace $X_{V}$ of $\operatorname{Spec}_{r}\left(k_{V}\right)$ called the push-down of $X$, and conversely, given a subspace of orderings $Y$ in $k_{V}$, the set ${ }^{V} Y$ of orderings of $K$ which are compatible with $V$ and specialize to an ordering of $Y$ is a subspace, called the pull-back of $Y$. By Baer-Krull [BCR, Theorem 10.1.10], we have ${ }^{V} Y=\widehat{H} \times Y$, where $\widehat{H}=\operatorname{Hom}\left(\Gamma / 2 \Gamma, \mathbb{F}_{2}\right)$, so that that ${ }^{V} Y$ is the extension ${ }^{V} Y=Y[\Gamma / 2 \Gamma]$. We will use the notation $\sigma=(\widehat{h}, \gamma) \in X$, with $\gamma \in Y, \widehat{h} \in \widehat{H}$, and say that $\sigma$ specializes to $\gamma$. We will refer to the fiber $\widehat{H} \times\{\gamma\}$ as the set of generalizations of $\gamma$. The pull-back of a single ordering $\gamma \in \operatorname{Spec}_{r}\left(k_{V}\right)$ will be denoted by $F_{\gamma}$.

From now on we assume that $K$ is a finitely generated extension of $\mathbb{R}$ of transcendence degree $n$. In particular $K$ is the field of rational functions of some irreducible real algebraic variety $M$ and any such $M$ is called a model of $K$. It is well known that the topological dimension of any model $M$ coincides with the transcendence degree of $K$ over $\mathbb{R}$. Another useful fact is that we can always find compact models of $K$. This is immediate by taking the projective closure of any given model or considering its one-point compactification, which is always possible in the real case; cf. [BCR, section 5.3].

Definition 1.1. Let $(X, G)$ be a finite subspace of orderings of $\Sigma_{K}=\operatorname{Spec}_{r}(K)$. We define the notion of $(X, G)$ to be geometric by induction along its tree:

1) The atomic space $E=\{\sigma\}$ is geometric if the convex hull $W_{\sigma}$ of $\mathbb{R}$ in $K$ with respect to $\sigma$ is a discrete valuation ring of rank $n$.

2) If $X=X_{1}+X_{2}$, then $X$ is geometric if and only if $X_{1}$ and $X_{2}$ are geometric. 
3) If $X=X_{1}[H]$ is an extension with \# $(H)=2^{\alpha}$, then $X$ is geometric if there is a discrete valuation ring $V$ of $K$ of rank $\alpha$ and residue field $k_{V}$ finitely generated over $\mathbb{R}$ with transcendence degree $n-\alpha$, such that $X_{1}$ is a geometric subspace of $\operatorname{Spec}_{r}\left(k_{V}\right)$ and $X$ is the pull-back of $X_{1}$ by $V$.

Remark 1.2. Let us denote by $\mathcal{B}_{X}$ the family of all valuation rings of $K$ compatible with some element of $X$. It follows from the definition that if $X$ is geometric, then all the valuations of $\mathcal{B}_{X}$ are discrete of rank $n$ and with finitely generated residue field. In fact this can be taken as an alternative definition of GSO.

Definition 1.3. We say that a finite geometric subspace of orderings $X$ is fully realized in an open semialgebraic subset $S$ of a compact model $M$ of $K$ if any $\sigma \in X$ specializes to a point in $S$ and its chain of specializations in $\operatorname{Spec}_{r}(\mathcal{R}(M))$ has length $n$, i.e. there are $\sigma^{(i)} \in \operatorname{Spec}_{r}(\mathcal{R}(M))$ such that $\sigma \rightarrow \sigma^{(1)} \rightarrow \cdots \rightarrow \sigma^{(n)}=\{x\} \in S$.

As a first result on the abundance of geometric spaces of orderings, we have:

Proposition 1.4. a) Any finite space of orderings of stability index $s$ is isomorphic to a geometric subspace realized in any open semialgebraic subset $S$ of dimension $s$ of a real algebraic variety $M$.

b) Let $X$ be a geometric space of orderings of $K$. Then there is a compact model of $K$ in which $X$ is fully realized. Moreover, for any compact model $M$ of $K$ there is sequence of blowings-up, $M_{r} \rightarrow M_{r-1} \rightarrow \cdots \rightarrow M_{0}=M$, such that $X$ is realized in $M_{r}$.

Proof. Part a) is [Br1, Proposition 3.3]. For part b) it is enough to blow-up $M$ till we reach a model in which all valuations (a finite number) of $\mathcal{B}_{X}$ have a center of dimension equal to the transcendence degree of their residue fields.

\section{Density of GeOMETRIC SPACES OF ORDERINGS}

Fix an integer $k \geq 0$. Any subspace $X$ of $\Sigma_{K}$ with $k$ elements can be seen as a $k$-tuple in the product $\Sigma^{k}=\Sigma \times \cdots \times \Sigma$ ( $k$ times). This identification is not bijective, unless we identify the tuples in $\Sigma^{k}$ up to permutations, but this technicality is irrelevant for our purposes. The set $\Sigma^{k}$ carries the product topology of the Harrison topology [AnBrRz, I.3.3] of each factor space and we can discuss approximation properties in it. We recall that two finite subspaces of orderings are isomorphic if and only if they have the same structure tree.

In this section we will show the following:

Theorem 2.1. Let $K$ be a finitely generated extension of $\mathbb{R}$ of transcendence degree $n$. Let $X \subset \Sigma_{K}$ be a subspace of orderings with $k$ elements. Then $X$ can be arbitrarily approximated in the Harrison topology by a geometric subspace $X^{\prime}$ isomorphic to it.

Before entering into the proof of the theorem we need a few technical results:

Lemma 2.2. Let $X \subset \Sigma$ be a subspace of orderings and assume that $X=X_{1}+X_{2}$. If $X_{1}^{\prime}$ and $X_{2}^{\prime}$ are close enough to $X_{1}$ and $X_{2}$, then $X_{1}^{\prime} \cup X_{2}^{\prime}=X_{1}^{\prime}+X_{2}^{\prime}$ as spaces of orderings.

Proof. By the 4-element fan criterion ([Mr5, Theorem 4.1.2], [AnBrRz, Theorem $7.2 \mathrm{c})]$ ), a union $X_{1} \cup X_{2}$ of two disjoint closed subsets is a sum if and only if any 4-element fan is contained either in $X_{1}$ or in $X_{2}$, which in turn can be translated 
in terms that any element of $X_{1}$ (resp. $X_{2}$ ) can be separated from any group of three elements of $X_{2}$ (resp. $X_{1}$ ). Now, this is an open condition in the Harrison topology, so that it is also satisfied by any $X_{1}^{\prime}$ and $X_{2}^{\prime}$ close enough to $X_{1}$ and $X_{2}$, and the lemma follows at once.

For the next result, given a field extension $K \subset L$ and a subspace of orderings $Y \subset \Sigma_{L}$ we denote by $Y \cap K$ the restriction of $Y$ to $K$, that is, $Y \cap K=\{\sigma \cap K \mid$ $\sigma \in Y\}$.

Lemma 2.3. Let $K \subset L$ be a field extension and let $Y \subset \Sigma_{L}$ be a finite subspace of orderings of $L$. Then there is a finitely generated extension $F, K \subset F \subset L$, such that $X=Y \cap F$ is isomorphic to $Y$.

Proof. We work by induction along the tree of $Y$. If $Y$ is atomic, the result is trivial. If $Y=Y_{1}+Y_{2}$, this means that $Y=Y_{1} \cup Y_{2}$ and any 4-element fan is contained either in $Y_{1}$ or $Y_{2}$. By induction there is a finitely generated extension of $K$ such that $X_{i}=Y_{i} \cap F$ is isomorphic to $Y_{i}$. Moreover, adding, if necessary, more elements to $F$, we may assume that $F$ contains functions which separate each element of $Y_{1}$ from each group of three of $Y_{2}$ and conversely. Thus, $X=X_{1} \cup X_{2}=X_{1}+X_{2}$ and $X$ is isomorphic to $Y$.

Finally, let us assume that $Y$ is an extension, $Y=Y^{\prime}[H]$, where $Y^{\prime}$ is not an extension. Let $V$ be the smallest valuation ring of $L$ compatible with $Y$. Then $Y^{\prime} \approx Y_{V}$, the push-down of $Y, H$ is isomorphic to a subgroup of $\Gamma_{V} / 2 \Gamma_{V}$ and $Y$ is a subspace of the pull-back ${ }^{V} Y_{V}=Y_{V}\left[\Gamma_{V} / 2 \Gamma_{V}\right]$. Now, let $W=V \cap K$. $W$ is a valuation ring of $K$ with $k_{W} \subset k_{V}$ and $\Gamma_{W} \subset \Gamma_{V}$. By the induction hypothesis there is a finitely generated extension $k_{1}$ of $k_{W}$ with $k_{1} \subset k_{V}$ and such that $X_{1}=Y^{\prime} \cap k_{1}$ is isomorphic to $Y^{\prime}$. Let $a_{1}, \ldots, a_{r} \in V$ be such that their classes in $k_{V}$ generate $k_{1}$ over $k_{W}$. Finally let $b_{1}, \ldots, b_{s} \in V$ be such that their values $v\left(b_{1}\right), \ldots, v\left(b_{s}\right)$ generate $H$ (remember that it is a finite group) and consider the extension $F=K\left(a_{1}, \ldots, a_{r}, b_{1}, \ldots, b_{s}\right)$. Then $V^{\prime}=V \cap F$ is a valuation ring such that $\Gamma_{V^{\prime}} / 2 \Gamma_{V^{\prime}}$ contains $H$ as a subgroup and $k_{1}$ is a subfield of $k_{V^{\prime}}$ and it follows at once that $Y \cap F$ is isomorphic to $Y$.

Proof of Theorem 2.1. Take a compact model $M$ of $K$. Then every regular function is bounded on $M$, so that every real valuation ring of $K$ contains the $\operatorname{ring} \mathcal{R}(M)$ of regular functions of $M$. Set $X=\left(\sigma_{1}, \ldots, \sigma_{k}\right)$ and let $U=U_{1} \times \cdots \times U_{k}$ be an open neighbourhood of $X$ in $\Sigma^{k}$, with $U_{i}=\left\{f_{i 1}>0, \ldots, f_{i r_{i}}>0\right\}, f_{i j} \in \mathcal{R}(M)$. After shrinking the $U_{i}$ 's we may assume that they are pairwise disjoint, and in particular the $f_{i j}$ 's separate the orderings of $X$. We work by induction on the tree of $X$.

If $X=\{\sigma\}$ (the atomic space), then $U=\left\{f_{1}>0, \ldots, f_{r}>0\right\}$. By the ArtinLang Theorem [BCR, Theorem 4.1.2], the semialgebraic set $S=\left\{f_{1}>0, \ldots, f_{r}>\right.$ $0\} \cap \operatorname{Reg}(M)$ is not empty. Take any point $x \in S$. Let $V$ be a discrete valuation ring of $K$ of rank $n$, dominating the regular local ring $\mathcal{R}(M)_{x}[\mathrm{AnBrRz}$, Lemma II.3.4], and let $\tau$ be any ordering compatible with $V$. Then $\tau$ is geometric, $\tau \in U$ and $X \approx\{\tau\}$.

If $X=X_{1}+X_{2}$, then, after reordering if necessary, $X_{1}=\left(\sigma_{1}, \ldots, \sigma_{\ell}\right) \subset \Sigma^{\ell}$, $X_{2}=\left(\sigma_{\ell+1}, \ldots, \sigma_{k}\right) \subset \Sigma^{k-\ell}$ and we have neighbourhoods $U^{(1)}=U_{1} \times \cdots \times U_{\ell}$ and $U^{(2)}=U_{\ell+1} \times \cdots \times U_{k}$ of $X_{1}$ and $X_{2}$ respectively. By induction we find $X_{1}^{\prime} \in U^{(1)}$ and $X_{2}^{\prime} \in U^{(2)}$ isomorphic to them and geometric on $K$. Moreover, restricting the neighbourhoods if necessary, by Lemma 2.2 above we have that 
$X^{\prime}=X_{1}^{\prime} \cup X_{2}^{\prime}=X_{1}^{\prime}+X_{2}^{\prime}$. Thus, $X^{\prime} \in U$ is geometric on $K$ and isomorphic to $X$, as wanted.

Next, let $X \approx X_{1}[H]$ be an extension with $\#(H)=2^{a}$ and $X_{1}$ not an extension. By convention $E\left[\mathbb{Z}_{2}\right]$ is represented as the sum $E+E$ and not as an extension, so that $\#\left(X_{1}\right) \geq 2$. We have $X=\widehat{H} \times X_{1}$. Let $\pi: X \rightarrow X_{1}$ be the canonical projection and set $X_{1}=\left\{\gamma_{0}, \ldots, \gamma_{m}\right\}, \widehat{H}=\left\{\hat{h}_{0}, \ldots, \hat{h}_{2^{a}-1}\right\}$. We order the elements of $X$ in a matrix arrangement:

$\begin{array}{llll}\sigma_{0}^{0} & \sigma_{0}^{1} & \ldots & \sigma_{0}^{m} \\ \sigma_{1}^{0} & \sigma_{1}^{1} & \ldots & \sigma_{1}^{m} \\ \vdots & \vdots & & \vdots \\ \sigma_{2^{a}-1}^{0} & \sigma_{2^{a}-1}^{1} & \ldots & \sigma_{2^{a}-1}^{m}\end{array}$

where $\sigma_{j}^{i}=\left(\hat{h}_{j}, \gamma_{i}\right)$. Let $W$ be the minimal valuation ring compatible with $X$ and let $\Gamma$ be its value group. Let $X_{W}$ be the push-down of $X$ by $W$. Then $X_{1} \approx X_{W}$ and $X=X_{1}[H]$ is a subspace of ${ }^{W} X_{W}=X_{W}[\Gamma / 2 \Gamma]$; cf. [AnBrRz, Proposition IV.2.13]. It follows at once that $r=\operatorname{dim}_{\mathbb{F}_{2}} \Gamma / 2 \Gamma \geq a$. From now on we identify $X_{1}$ and $X_{W}$.

Now we apply resolution of singularities I and II ([Hk]), so that after finitely many blowings-up we may assume that $M$ is nonsingular and all the $f_{i j}$ 's are normal crossings. Let $\mathfrak{p}=\mathfrak{m}_{W} \cap \mathcal{R}(M)$ be the center of $W$ in $\mathcal{R}(M)$ where $\mathfrak{m}_{W}$ is the maximal ideal of $W$. Then $A=\mathcal{R}(M)_{\mathfrak{p}}$ is a regular local ring of dimension, say, $d$, and has a regular system of parameters $x_{1}, \ldots, x_{d}$ such that, for all $i, j$,

$$
f_{i j}=u_{i j} x_{1}^{\alpha_{i j 1}} \cdots x_{d}^{\alpha_{i j d}}
$$

where the $u_{i j}$ are units of $A$ and the $\alpha_{i j k}$ are nonnegative integers.

In this situation the quotient field $\kappa(\mathfrak{p})$ of $A / \mathfrak{p}$ is a subfield of the residue field $k_{W}$ of $W$ and performing some extra blowings-up we may assume also, by Lemma 2.3 , that $\mathcal{R}(M)$ contains enough functions so that the restrictions $\gamma_{i} \cap \kappa(\mathfrak{p})$ form, in $\operatorname{Spec}_{r}(\kappa(\mathfrak{p}))$, a subspace isomorphic to $X_{1}$, which we still denote by $X_{1}$.

Next, notice that the signs of the elements $f_{i j}$ in any ordering $\sigma \rightarrow \gamma_{i}$ are completely determined by the signs of the parameters $x_{l}$ in $\sigma$ and the signs of the units (or more properly of their residue classes) in $\gamma_{i}$. Fix one of the $\gamma_{i}$, say $i=0$. Thus, if we associate to each ordering $\sigma \in X$ the $d$-tuple $\left(\sigma\left(x_{1}\right), \ldots, \sigma\left(x_{d}\right)\right)$, these $d$-tuples cannot coincide on two orderings over $\gamma_{0}$. In particular we get that $2^{d} \geq 2^{a}$, i.e. $a \leq d$. We will see that after some operations which in the end will be quadratic transforms of $A$ (and hence of $M$ ) we will find a system of parameters $x_{1}, \ldots, x_{a}, x_{a+1}, \ldots, x_{d}$ such that $X$ will coincide with the product $\widehat{G} \times X_{1}$, where $\widehat{G}$ is the subspace of the mappings $\varepsilon$ of $\left\{x_{1}, \ldots, x_{d}\right\}$ into $\mathbb{Z}_{2}=\{-1,+1\}$ such that $\varepsilon\left(x_{\ell}\right)=+1$ for all $\ell>a$.

To make this precise let us come back to our matricial distribution of the elements of $X$. Take any ordering over $\gamma_{0}$, say $\sigma_{0}^{0}$. Changing $x_{j}$ by $-x_{j}$ if necessary, we may assume that $\sigma_{0}^{0}\left(x_{j}\right)=1$ for all $j$ (this $\sigma_{0}^{0}$ will define the zero homomorphism of $\left\{x_{1}, \ldots, x_{d}\right\}$ into $\left.\mathbb{Z}_{2}\right)$. Now take $a$ more elements over $\gamma_{0}$, say $\sigma_{1}^{0}, \ldots, \sigma_{a}^{0}$ which are linearly independent (and therefore $\sigma_{0}^{0}, \sigma_{1}^{0}, \ldots, \sigma_{a}^{0}$ generate the fiber over $\gamma_{0}$ ). Moreover, now take an element in each of the other columns, say $\sigma_{0}^{1}, \ldots, \sigma_{0}^{m}$. Notice that the elements $\sigma_{0}^{0}, \ldots, \sigma_{a}^{0}, \sigma_{0}^{1}, \ldots, \sigma_{0}^{m}$ generate $X$, since they generate the first column and with this and the selected elements in each of the other columns we can get any element of $X$ by completing squares of 4 -element fans: $\sigma_{j}^{i}=\sigma_{j}^{0} \sigma_{0}^{i} \sigma_{0}^{0}$. 
Claim. After some additional blowings-up, we find a regular local ring $B$ dominating $A$, with the same residue field and a system of parameters $y_{1}, \ldots, y_{d}$ of $B$ such that all $f_{i j}$ 's are normal crossings in $B$ with respect to them and for all $j=0, \ldots, a$

$$
\sigma_{j}^{0}\left(y_{k}\right)= \begin{cases}+1 & \text { for } k \geq j+1 \\ -1 & \text { if } k=j .\end{cases}
$$

In other words, we are saying that the matrix of signs of the $\sigma_{j}^{0}, j=1, \ldots, a$, at the system of parameters is lower triangular with -1 in the main diagonal, i.e. we have an arrangement of the type:

\begin{tabular}{c|cccccc|cccc} 
& $y_{1}$ & $y_{2}$ & $y_{3}$ & $\cdots$ & $y_{a-1}$ & $y_{a}$ & $y_{a+1}$ & $y_{a+2}$ & $\cdots$ & $y_{d}$ \\
\hline$\sigma_{0}^{0}$ & 1 & 1 & 1 & $\cdots$ & 1 & 1 & 1 & 1 & $\cdots$ & 1 \\
\hline$\sigma_{1}^{0}$ & -1 & 1 & 1 & $\cdots$ & 1 & 1 & 1 & 1 & $\cdots$ & 1 \\
$\sigma_{2}^{0}$ & $*$ & -1 & 1 & $\cdots$ & 1 & 1 & 1 & 1 & $\cdots$ & 1 \\
$\vdots$ & $\vdots$ & $\vdots$ & $\vdots$ & & $\vdots$ & $\vdots$ & $\vdots$ & $\vdots$ & & $\vdots$ \\
$\sigma_{a-1}^{0}$ & $*$ & $*$ & $*$ & $\cdots$ & -1 & 1 & 1 & 1 & $\cdots$ & 1 \\
$\sigma_{a}^{0}$ & $*$ & $*$ & $*$ & $\cdots$ & $*$ & -1 & 1 & 1 & $\cdots$ & 1 \\
\hline
\end{tabular}

where $*$ represents +1 or -1 .

In fact, taking into account that the functions $f_{i j}$ separate the orderings of $F_{\gamma_{0}}$ and that $\sigma_{0}^{0}, \sigma_{1}^{0}, \ldots, \sigma_{a}^{0}$ generate this set, they must go to independent vectors under the map

defined by

$$
\varphi: \sigma_{0}^{0} F_{\gamma_{0}} \longrightarrow \mathbb{Z}_{2}^{m}
$$

$$
\sigma_{0}^{0} \sigma \longmapsto\left(\sigma\left(x_{1}\right), \ldots, \sigma\left(x_{d}\right)\right) .
$$

The proof of the claim consists of translating to our situation the classical Gauss elimination algorithm to make lower triangular the matrix of signs of the images of the $\sigma$ 's by these mappings. Remember that we use the multiplicative structure on $\mathbb{Z}_{2}$ so that taking the difference between columns $k$ and $\ell$ of the matrix corresponds to taking the quotient of the column $k$ over column $\ell$. This, in turn, replaces the parameter $x_{k}$ by $x_{k} / x_{\ell}$, which corresponds to performing a blowing-up in $A$.

Let us see it formally. We work by induction on $\ell=0,1, \ldots$. Assume that we have already found a local regular ring $A^{(\ell)}$ dominating $A$ with the same residue field as the latter and with a system of parameters $x_{1}^{(\ell)}, \ldots, x_{d}^{(\ell)}$ such that the $f_{i j}$ are normal crossings for them in $A^{(\ell)}$ and for all $0 \leq j \leq \ell$ we have $\sigma_{j}^{0}\left(x_{k}^{(\ell)}\right)=1$ for all $k \geq j+1$ and $\sigma_{j}^{0}\left(x_{j}^{(\ell)}\right)=-1$. Notice that for $\ell=0, A^{(0)}=A$ fulfills this condition trivially. We construct $A^{(\ell+1)}$ as follows:

Consider $\sigma_{\ell+1}^{0}$. There is $k \geq \ell+1$ such that $\sigma_{\ell+1}^{0}\left(x_{k}^{(\ell)}\right)=-1$, for otherwise the above table of signs shows that $\sigma_{0}^{0} \sigma_{\ell+1}^{0}$ would be in the subspace generated by $\sigma_{0}^{0} \sigma_{1}^{0}, \ldots, \sigma_{0}^{0} \sigma_{\ell}^{0}$, against our assumption that $\sigma_{0}^{0}, \ldots, \sigma_{a}^{0}$ are affine independent. Then, after reordering $x_{\ell+1}^{(\ell)}, \ldots, x_{d}^{(\ell)}$, we may assume that $\sigma_{\ell+1}^{0}\left(x_{k}^{(\ell)}\right)=1$ for $k \geq r$ and $\sigma_{\ell+1}^{0}\left(x_{k}^{(\ell)}\right)=-1$ for $\ell+1 \leq k<r$. Consider the extension

$$
A^{(\ell+1)}=A^{(\ell)}\left[x_{\ell+2}^{(\ell)} / x_{\ell+1}^{(\ell)}, \ldots, x_{r-1}^{(\ell)} / x_{\ell+1}^{(\ell)}\right]_{\left(x_{1}^{(\ell)}, \ldots, x_{\ell+1}^{(\ell)}, x_{\ell+2}^{(\ell)} / x_{\ell+1}^{(\ell)}, \ldots, x_{r-1}^{(\ell)} / x_{\ell+1}^{(\ell)}, x_{r}^{(\ell)}, \ldots, x_{d}^{(\ell)}\right)}
$$

and set $x_{k}^{(\ell+1)}=x_{k}^{(\ell)}$ for $1 \leq k \leq \ell+1, x_{k}^{(\ell+1)}=x_{k}^{(\ell)} / x_{\ell+1}^{(\ell)}$ for $\ell+2 \leq k \leq r-1$ and $x_{k}^{(\ell+1)}=x_{k}^{(\ell)}$ for $k \geq r$. An immediate computation shows that for $1 \leq j \leq \ell+1$ 
we have $\sigma_{j}^{0}\left(x_{k}^{(\ell+1)}\right)=1$ for all $k \geq j+1$ and $\sigma_{j}^{0}\left(x_{j}^{(\ell+1)}\right)=-1$, so that we have done the step $\ell+1$. This shows the claim.

Now, let us go back to the matrix representation of $X$ and notice that the signs of the parameters $y_{a+1}, \ldots, y_{d}$ produce a partition of $X$ which is compatible with the columns. In fact, suppose that $\sigma_{0}^{i}\left(y_{k}\right)=\varepsilon$ for some $k=a+1, \ldots, d$, where $\varepsilon \in\{1,-1\}$. Then for any other $j=1, \ldots, 2^{a}-1$ we have $\sigma_{j}^{i}\left(y_{k}\right)=$ $\sigma_{0}^{0}\left(y_{k}\right) \sigma_{0}^{i}\left(y_{k}\right) \sigma_{j}^{0}\left(y_{k}\right)=\sigma_{0}^{i}\left(y_{k}\right)=\varepsilon$; in other words all members of the column $i$ have the same sign at any of the $y_{k}$ for $k=a+1, \ldots, d$. In particular, the family $S_{\varepsilon}=\left\{\varepsilon_{a+1} y_{a+1}>0, \ldots, \varepsilon_{d} y_{d}>0\right\}$, where $\varepsilon=\left(\varepsilon_{a+1}, \ldots, \varepsilon_{d}\right) \in\{-1,1\}^{d-a}$, produces a partition of $X$ compatible with the columns.

Taking into account the structure of $X=X_{1}[H]$ as extension, this means that for each $k>a$ the function $y_{k}$ defines a function over $X_{1}$. Setting $X_{1, \varepsilon}=X_{1} \cap S_{\varepsilon}$ we have a partition $X_{1}=\bigcup_{\varepsilon}\left\{X_{1, \varepsilon}\right\}$ of $X_{1}$ into disjoint basic sets.

Now we consider the diagram

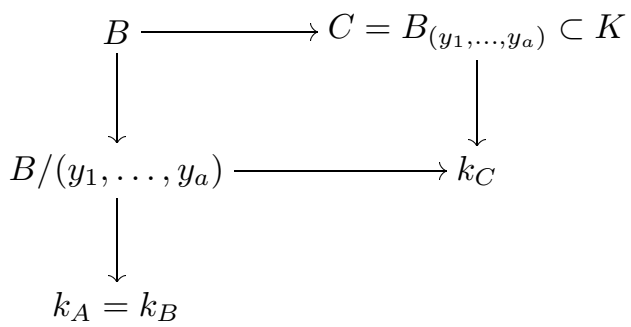

where $k_{B}, k_{C}$ stand for the residue fields of $B$ and $C$ respectively. By construction, these two residue fields are finitely generated over $\mathbb{R}$. Now let $k_{B}^{\prime}$ be a quasicoefficient field of $B$, that is, a subfield $k_{B}^{\prime} \subset B$, such that the extension $k_{B}^{\prime} \subset k_{B}$ induced by the canonical homomorphism $B \rightarrow k_{B}$ is algebraic (even finite in our case). Then, since $k_{C}$ is the quotient field of the ring $B /\left(y_{1}, \ldots, y_{a}\right)$, which is local regular of dimension $d-a$ and $k_{B}=k_{A}=\kappa(\mathfrak{p})$, we get

$$
\begin{aligned}
& \operatorname{tr.deg} .\left[k_{C}: \mathbb{R}\right]=\operatorname{tr} . \operatorname{deg} \cdot\left[k_{C}: k_{B}^{\prime}\right]+\operatorname{tr} . \operatorname{deg} \cdot\left[k_{B}^{\prime}: \mathbb{R}\right] \\
& \quad \geq d-a+\operatorname{tr} \cdot \operatorname{deg} \cdot[\kappa(\mathfrak{p}): \mathbb{R}]=d-a+\operatorname{dim}(\mathcal{R}(M) / \mathfrak{p}) \\
& \quad=d-a+\operatorname{dim}(\mathcal{R}(M))-\operatorname{ht}(\mathfrak{p})=d-a+n-d=n-a .
\end{aligned}
$$

Let us lift our space $X_{1}$ from $k_{B}=\kappa(\mathfrak{p})$ to $k_{C}$. Take $\gamma_{i} \in X_{1}$, and assume that $\gamma_{i} \in S_{\varepsilon}$, i.e., $\gamma_{i}\left(y_{k}\right)=\varepsilon_{k}$ for any $k=a+1, \ldots, d$. Since $B /\left(y_{1}, \ldots, y_{a}\right)$ is a local regular ring with parameters $y_{a+1}, \ldots, y_{d}$, we can lift $\gamma_{i}$ to a unique ordering $\tau_{i}$ of $k_{C}$ such that $\gamma_{i}\left(y_{j}\right)=\tau_{i}\left(y_{j}\right)=\varepsilon_{k}$ for $a+1 \leq j \leq d$.

We claim that $Y_{1}=\left\{\tau_{1}, \ldots, \tau_{m}\right\}$ is a subspace of orderings of the extension $\widehat{G} \times X_{1}$ where $\widehat{G}$ is the group of mappings of $\left\{y_{a+1}, \ldots, y_{d}\right\}$ into $\mathbb{Z}_{2}$, and that $Y_{1} \approx X_{1}$. Indeed, notice that as element of $\widehat{G} \times X_{1}$ we have $\tau_{i}=\left(h, \gamma_{i}\right)$, where $h$ is the function such that $h\left(y_{k}\right)=\varepsilon_{k}=\gamma_{i}\left(y_{k}\right)$ for $k=a+1, \ldots, d$. To check that it is a subspace we have to see that for any four-element fan $F \subset \widehat{G} \times X_{1}$, $\#\left(F \cap Y_{1}\right) \neq 3$; cf. [AnBrRz, Corollary IV.1.8]. Assume that $F=\left\{\left(g_{i}, \alpha_{i}\right)\right\}_{1 \leq i \leq 4}$, with, say $\left(g_{1}, \alpha_{1}\right),\left(g_{2}, \alpha_{2}\right),\left(g_{3}, \alpha_{3}\right) \in Y_{1}$. Then $F_{1}=\left\{\alpha_{i}\right\}_{1 \leq i \leq 4}$ is a fan in $X_{1}$, i.e., $\alpha_{1} \alpha_{2} \alpha_{3}=\alpha_{4}$. Thus,

$$
g_{4}\left(y_{q}\right)=g_{1}\left(y_{q}\right) g_{2}\left(y_{q}\right) g_{3}\left(y_{q}\right)=\alpha_{1}\left(y_{q}\right) \alpha_{2}\left(y_{q}\right) \alpha_{3}\left(y_{q}\right)=\alpha_{4}\left(y_{q}\right),
$$

which means that $\left(g_{4}, \alpha_{4}\right) \in Y_{1}$ as wanted. Clearly $Y_{1} \approx X_{1}$. 
To finish, by the induction hypothesis, there is a geometric subspace $X_{1}^{\prime}$ of $\operatorname{Spec}_{r}\left(k_{C}\right)$ isomorphic to $Y_{1}$ and close enough to it so that each element $\gamma_{i}^{\prime} \in X_{1}^{\prime}$ satisfies $\gamma_{i}^{\prime}\left(y_{k}\right)=\tau_{i}\left(y_{k}\right)=\gamma_{i}\left(y_{k}\right)$ for all $k=a+1, \ldots, d$ and also $\gamma_{i}^{\prime}\left(u_{r t}\right)=\tau_{i}\left(u_{r t}\right)=$ $\gamma_{i}\left(u_{r t}\right)$ on the units $u_{r t}$ coming from the functions $f_{r t}$.

Since $C$ is local regular with parameters $y_{1}, \ldots, y_{a}$, we can produce an extension $X^{\prime}=\widehat{H} \times X_{1}^{\prime}$ with $\widehat{H}$ equal to the set of mappings of $\left\{y_{1}, \ldots, y_{a}\right\}$ into $\mathbb{Z}_{2}$ using a discrete valuation ring of rank $a$ which dominates $C$. By construction $X^{\prime}$ is geometric and $X^{\prime} \approx X$. Moreover, if we set $\beta_{j}^{i}=\left(h_{j}, \gamma_{i}^{\prime}\right)$, where $h_{j} \in \widehat{H}$ is the function defined by $h_{j}\left(y_{k}\right)=\sigma_{j}^{0}\left(y_{k}\right)$ for $1 \leq k \leq a$, we have that $\beta_{j}^{i}\left(f_{r t}\right)=\sigma_{j}^{i}\left(f_{r t}\right)$ for all $i, j, r, t$, and since the $f_{r t}$ 's define the neighbourhood $U \subset \Sigma_{K}$ of $X$ fixed at the beginning, we conclude $X^{\prime} \subset U$, which completes the proof.

As a consequence we have the following result. Remember that two constructible subsets $\widetilde{A}$ and $\widetilde{B}$ of $\Sigma_{K}$ are called separable if there exists $g \in K$ such that $\widetilde{A} \subset\{g>0\}$ and $\widetilde{B} \subset\{g<0\}$ :

Theorem 2.4. Let $K$ be a finitely generated extension of $\mathbb{R}$ of transcendence degree $n$. Let $\widetilde{A}$ and $\widetilde{B}$ be two disjoint constructible subsets of $\Sigma_{K}$. Then $\widetilde{A}$ and $\widetilde{B}$ are separable if and only if for any finite GSO $Y \subset \Sigma_{K}$ of chain-length $\leq 2^{n-1}, \widetilde{A} \cap Y$ and $\widetilde{B} \cap Y$ are separable in $Y$.

Proof. One direction is trivial by Bröcker's theorem ([Br3], [AnBrRz, Theorem, IV.7.12]). For the other, suppose that $\widetilde{A}$ and $\widetilde{B}$ are not separable. Then, again by Bröcker's theorem there exists a finite subspace $Y$ of $\Sigma_{K}$ such that $\widetilde{A} \cap Y$ and $\widetilde{B} \cap Y$ are not separable. Suppose that $\#(Y)=k$ and order its elements as a $k$-tuple $\left(\sigma_{1}, \ldots, \sigma_{k}\right)$. Also $Y$ has a finite number of different functions, namely the elements of the group $G_{Y}=K / Y^{\perp}$. Take $f_{1}, \ldots, f_{N} \in K$ such that

$$
G_{Y}=\left\{1 Y^{\perp},-1 Y^{\perp}, f_{1} Y^{\perp}, \ldots, f_{N} Y^{\perp}\right\},
$$

and let $f_{N+1}, \ldots, f_{r}$ be a family of elements of $K$ which describes the sets $A$ and $B$. Then, for any $j=1, \ldots, k$ consider the neighbourhood of $\sigma_{j}$ :

$$
U_{j}=\left\{f_{i}\left(\sigma_{j}\right) f_{i}>0 \mid i=1, \ldots, r\right\}
$$

and set $U=U_{1} \times \cdots \times U_{k}$. Thus $U$ is a neighbourhood of $Y$ in $\Sigma_{K}^{k}$ and by the density Theorem 2.1, there is a GSO, $X=\left(\tau_{1}, \ldots, \tau_{k}\right) \in U$, isomorphic to $Y$.

Notice that by our construction $\sigma_{j} \in A$ if and only if $\tau_{j} \in A$ and

$$
G_{X}=\left\{1 X^{\perp},-1 X^{\perp}, f_{1} X^{\perp}, \ldots, f_{N} X^{\perp}\right\} .
$$

Indeed, since $X$ and $Y$ are isomorphic we have $\#\left(G_{X}\right)=\#\left(G_{Y}\right)$. On the other hand, by construction all $f_{j}$ are nontrivial and different on $X$, so that they fill the whole $G_{X}$. Since the $f_{j}$ 's have the same signs over the $\sigma_{s}$ 's and the $\tau_{s}$ 's, we have that $A \cap X$ and $B \cap X$ are not separable.

\section{Generic Separation AND Walls}

Now let $M$ be an irreducible algebraic variety over $\mathbb{R}$ (which we assume compact), let $\mathcal{R}(M)$ be the ring of regular functions on $M$ and $\mathcal{K}(M)$ its quotient field. We denote by $\Sigma_{\mathcal{K}(M)}$ or simply by $\Sigma_{M}$ the space of orderings of $\mathcal{K}(M)$. Let $A, B$ be disjoint semialgebraic subsets of $M$. Recall that $A$ and $B$ are generically separable if and only if $\widetilde{A}$ and $\widetilde{B}$ are separable in $\Sigma_{M}$. 
Definition 3.1. a) We say that $A$ and $B$ are separable if there exists a regular function $f \in \mathcal{R}(M)$ such that $f(A)>0$ and $f(B)<0$.

b) We say that $A$ and $B$ are generically separable if there exists a nonzero regular function $f \in \mathcal{R}(M)$ such that $f(A) \geq 0$ and $f(B) \leq 0$. Equivalently, $A$ and $B$ are generically separable if there exists a proper algebraic subset $Y \subset M$ such that $A \backslash Y$ and $B \backslash Y$ are separable.

From now till Section 6 we will be dealing with the generic separation of $A$ and $B$. Since this is a birational property, we may assume that $M$ is nonsingular and that $A$ and $B$ are open. We are interested in testing the generic separation of $A$ and $B$ in terms of their boundaries. To start with, recall that if $\bar{A} \cap \bar{B}=\emptyset$, then $A$ and $B$ are separable (since $M$ is compact). So, only the case in which $A$ and $B$ have some common boundary is interesting.

Definition 3.2. We define the walls of $A$ and $B$ in $M$ as the irreducible $(n-1)$ dimensional components of the Zariski closure of the boundaries of $A$ and $B$, that is, the irreducible components of $\overline{\partial A}^{Z} \cup \overline{\partial B}^{Z}$ of dimension $n-1$.

Notice that since we are assuming that $M$ is nonsingular, for any wall $W$, the localization $\mathcal{R}(M)_{\mathcal{J}(W)}$ of the ring of regular functions on $M$ at the prime ideal defined by $W$ is a rank 1 , discrete, valuation ring of $K$ with residue field $\mathcal{K}(W)$. Hence, the pull-back of the space of orderings, $\Sigma_{W}$, of $\mathcal{K}(W)$ by the valuation $\mathcal{R}(M)_{\mathcal{J}(W)}$ is a subspace of orderings of $\Sigma_{K}$, isomorphic to the extension $\Sigma_{W}\left[\mathbb{Z}_{2}\right]$. From now on we fix the notation $\widehat{\mathbb{Z}}_{2}=\{1, i\}$, where 1 stands for the trivial map from $\mathbb{Z}_{2}$ to $\mathbb{Z}_{2}$ and $i$ for the identity. In particular for any $\gamma \in \Sigma_{W}$ we have two generalizations, $\alpha$ and $i \alpha$, in $\Sigma_{W}\left[\mathbb{Z}_{2}\right]$. Also, given a subset $\widetilde{C} \subset \Sigma_{W}\left[\mathbb{Z}_{2}\right]$ we denote by $\widetilde{C}_{W}$ the set of its specializations in $\Sigma_{W}$. From a geometric point of view, the space $\Sigma_{W}\left[\mathbb{Z}_{2}\right]$ represents a kind of germ of $M$ along $W$. As a first result we have (compare with $[\mathrm{AcBgFo}, \S 1]$ ):

Proposition 3.3. $A$ and $B$ are generically separable if and only if, for any nonsingular compact model $M$ of $K$ and any wall $W$ of $A$ and $B$ in $M, \widetilde{A}$ and $\widetilde{B}$ are separable in $\Sigma_{W}\left[\mathbb{Z}_{2}\right]$.

Proof. If $A$ and $B$ are generically separable, $\widetilde{A}$ and $\widetilde{B}$ are separable in $\Sigma_{K}$, and therefore so are they in any subspace $\Sigma_{W}\left[\mathbb{Z}_{2}\right]$. Conversely, assume that $A$ and $B$ are not generically separable. Then there exists a finite subspace of orderings $X \subset \Sigma_{K}$ such that $\widetilde{A} \cap X$ and $\widetilde{B} \cap X$ are not separable. Take $X$ with minimal cardinality. By Theorem 2.1 we may assume that $X$ is a GSO. Furthermore, if $X$ is a sum $X=X_{1}+X_{2}$, we have that $\widetilde{A} \cap X$ and $\widetilde{B} \cap X$ are separable if and only if $\widetilde{A} \cap X_{i}$ and $\widetilde{B} \cap X_{i}$ are separable for $i=1,2$. Thus, by the minimality assumption we get that $X$ is an extension, say $X=Y\left[\mathbb{Z}_{2}\right]$, with $Y$ a geometric space of orderings. Take a model $N$ of $K$ in which $X$ is fully realized; cf. Proposition $1.4 \mathrm{~b}$ ). Then $Y$ is a GSO of a hypersurface $W$ of $N$ and we claim that $W$ is a wall of $A$ and $B$ in $N$.

Indeed, suppose that $W \not \subset \overline{\partial A}^{Z} \cup \overline{\partial B}^{Z}$. Since we are assuming that $\widetilde{A}$ and $\widetilde{B}$ are not separated in $X$, we have that $\widetilde{A} \cap X \neq \emptyset, \widetilde{B} \cap X \neq \emptyset$ and since any $\sigma \in X$ specializes to an ordering of $Y$, we get in particular that $W \cap \bar{A} \neq \emptyset$ and $W \cap \bar{B} \neq \emptyset$. Now, since $W$ is not in the boundary of $A$, for any $\alpha \in \widetilde{A} \cap X$ we also have $i \alpha \in \widetilde{A} \cap X$ and the same happens for $B$. Therefore $\widetilde{A} \cap X$ and 
$\widetilde{B} \cap X$ are saturated for the specialization map $X \rightarrow Y$. This shows that $\widetilde{A} \cap X$ and $\widetilde{B} \cap X$ are separable if and only if their specializations $\widetilde{A}_{W}$ and $\widetilde{B}_{W}$ are separable in $Y$, so that, in particular, these are not separable. Finally consider the subspace $\{1\} \times Y \subset X$. The specialization map $X \rightarrow Y$ defines an isomorphism from $\{1\} \times Y$ onto $Y$ sending $\widetilde{A} \cap(\{1\} \times Y)$ to $\widetilde{A}_{W} \cap Y$ and $\widetilde{B} \cap(\{1\} \times Y)$ to $\widetilde{B}_{W} \cap Y$. This shows that $\widetilde{A} \cap(\{1\} \times Y)$ and $\widetilde{B} \cap(\{1\} \times Y)$ are not separable, against our assumption on the minimality of $X$.

Remark 3.4. The consideration of all models is important. For instance, going back to the example considered in Figure 2 we see that $A$ and $B$ are not generically separable, but they are separable in all spaces $\Sigma_{W}\left[\mathbb{Z}_{2}\right]$ for the walls $W$ in the picture of the left. The wall that produces the obstruction is the $y$-axis of the picture on the right, which only appears after blowing-up.

Looking for a more geometrical description of the obstructions to the separation we introduce the following definition, where $M, A$ and $B$ are as above:

Definition 3.5. We say that a wall $W$ is odd if there are $A^{\prime} \subset A, B^{\prime} \subset B$ which are generically separable and such that any function $f$ separating $A^{\prime}$ and $B^{\prime}$ changes sign across $W$. Similarly, we say that a wall $W$ is even if there are $A^{\prime} \subset A, B^{\prime} \subset B$ which are generically separable and such that any function $f$ separating $A^{\prime}$ and $B^{\prime}$ does not change sign across $W$.

In algebraic terms this means that if $t$ is a uniformizer of the local ring $\mathcal{R}(M)_{\mathcal{J}(W)}$, then, in this ring, we have $f=t^{m} u$ where $u$ is a unit and $m$ is odd (resp. even) if and only if $W$ is odd (resp. even). Here are some examples of odd and even walls (cf. also $[\mathrm{AcAnBg}]$ ):

Example 3.6. The wall $W$ is odd in Figure 3 where $A^{\prime}=A$ and $B^{\prime}=B$. However $W$ is even in Figure 4 where we also take $A^{\prime}=A$ and $B^{\prime}=B$.

Obviously, if some wall $W$ is simultaneously odd and even, then $A$ and $B$ are not generically separable. Figures 5 and 6 show two typical examples of this situation. Notice that in Figure 6, the four cubes $A$ on the left cause $W$ to be even, while the couple $A, B$ on the right make $W$ odd.

We want to show that the converse is also true: $A$ and $B$ are not generically separable if and only if there is a wall which is simultaneously odd and even, provided that we look at the walls in the appropriate model. But before explaining this, we need a new small detour into spaces of orderings.

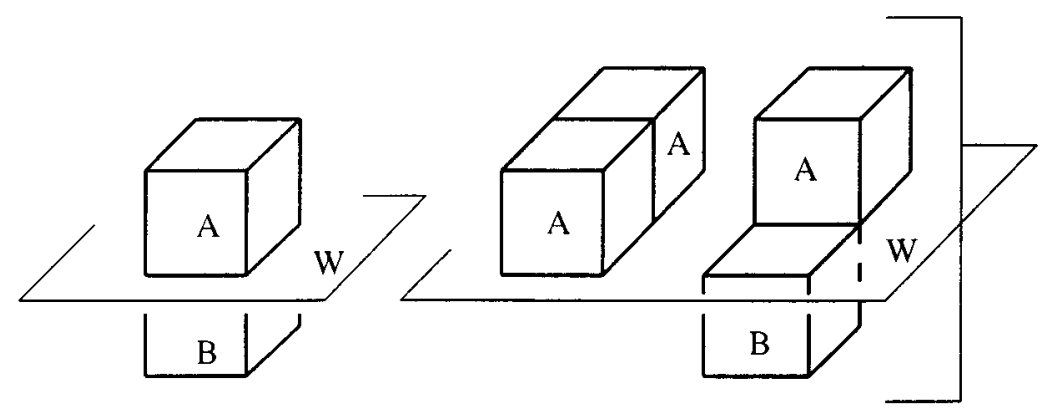

FigurE 3 


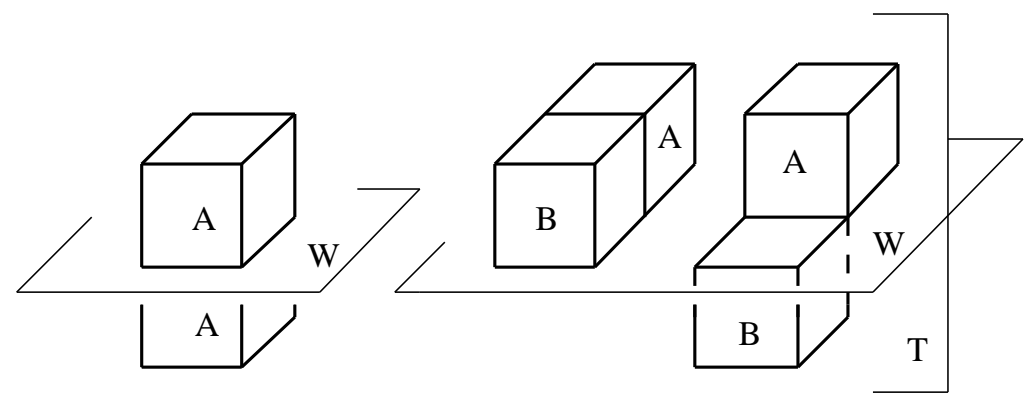

FiguRE 4

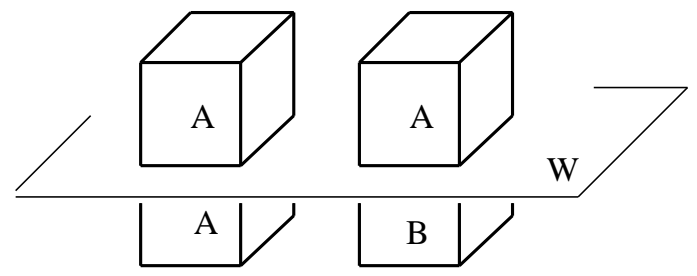

FigURE 5

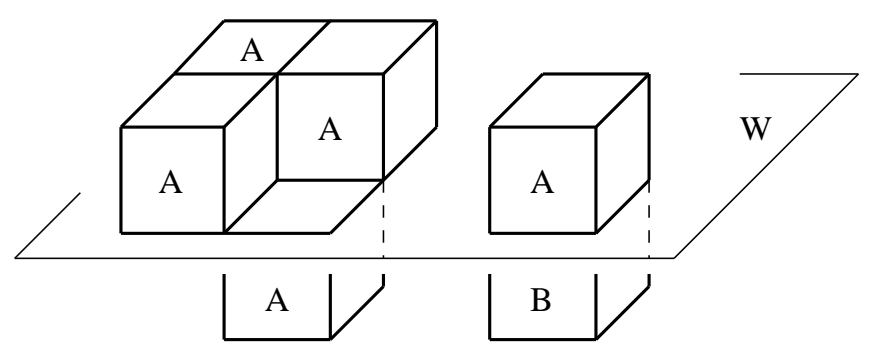

Figure 6

The notions of odd and even walls have a nice translation in terms of the space of orderings $\Sigma_{W}\left[\mathbb{Z}_{2}\right]$ which in fact give a criterion to recognize whether $W$ is odd or even. We define the saturations $\widetilde{A}^{\#}$ and $\widetilde{B} \widetilde{A}^{\#}$ of $\widetilde{A}$ and $\widetilde{B}$ in $\Sigma_{M}$ as:

$$
\begin{aligned}
& \widetilde{A}^{\#}=\left\{\sigma \in \Sigma_{K} \mid \sigma\right. \text { can be written as a product of } \\
& \widetilde{B}^{\#}=\left\{\sigma \in \Sigma_{K} \mid \sigma\right. \text { can be written as a product of } \\
& \text { elements of } \widetilde{A} \cup \widetilde{B} \text { with an odd number of them in } \widetilde{B} \text { \}. }
\end{aligned}
$$

Notice that $\sigma$ is always the product of an odd number of elements of $A \cup B$ (because $\sigma \in \Sigma_{K}$ ). Thus, in $\widetilde{A}^{\#}, \widetilde{B}^{\#}$ we are collecting all the orderings in which any function $f$ with $\left.f\right|_{\widetilde{A}}>0$ and $\left.f\right|_{\widetilde{B}}<0$ also satisfies $\left.f\right|_{\widetilde{A}^{\#}}>0$ and $\left.f\right|_{\widetilde{B}} \#<$. With this terminology Bröcker's separation theorem in spaces of orderings ([Br3, 
Theorem 2.1], [AnBrRz, Theorem IV.7.12]) can be restated by saying that $\widetilde{A}$ and $\widetilde{B}$ are separable if and only if $\widetilde{A}^{\#} \cap \widetilde{B}^{\#}=\emptyset$.

Next we translate the notion of odd and even walls into spaces of orderings. Notice that for any $\widetilde{A} \subset \Sigma_{W}\left[\mathbb{Z}_{2}\right]$ we have $\left(\widetilde{A}_{W}\right)^{\#}=\left(\widetilde{A}^{\#}\right)_{W}$.

Proposition 3.7. a) $W$ is odd if and only if there is $\sigma \in \Sigma_{W}\left[\mathbb{Z}_{2}\right]$ such that $\sigma \in \widetilde{A}$ and $i \sigma \in \widetilde{B}^{\#}$.

b) $W$ is even if and only if there is $\sigma \in \Sigma_{W}\left[\mathbb{Z}_{2}\right]$ such that $\sigma \in \widetilde{A}^{\#}$ and $i \sigma \in \widetilde{A} \#$ or $\sigma \in \widetilde{B}^{\#}$ and $i \sigma \in \widetilde{B}^{\#}$.

Proof. We only prove a), the proof of b) being analogous. So, suppose that $W$ is odd. Replacing $A$ and $B$ by the sets $A^{\prime}$ and $B^{\prime}$ stated in the definition of odd walls, we may assume that $A$ and $B$ are separable and that any function separating them changes sign at $W$. Let $f$ be any such function, say with $\left.f\right|_{A}>0,\left.f\right|_{B}<0$. In particular $f$ vanishes on $W$. If $\widetilde{A}_{W} \cap \widetilde{B}_{W} \neq \emptyset$, then a) follows at once. So, suppose that $\widetilde{A}_{W} \cap \widetilde{B}_{W}=\emptyset$. Assume that $\widetilde{A}_{W}$ and $\widetilde{B}_{W}$ are separable, say $\left.g\right|_{\widetilde{A}_{W}}>0$, $\left.g\right|_{\widetilde{B}_{W}}<0$, where $g \in \mathcal{R}(M)$ is a unit in $\mathcal{R}(M)_{\mathcal{J}(W)}$. Let us see that $g$ separates $A$ and $B$ in a small neighbourhood of $W$, contradicting the hypothesis that $W$ is odd.

Indeed, consider the sets $\{g>0\}$ and $\{g<0\}$. We claim that the closure of the open semialgebraic set $T=(\{g>0\} \cap B) \cup(\{g<0\} \cap A)$ intersects $W$ into a subset of codimension at least one, or in other words, that this closure does not contain any open set of the regular locus of $W$. For, otherwise, there would be an ordering $\tau \in \widetilde{T}$ which would specialize to an ordering $\gamma \in \Sigma_{W}$. Now, if $\tau \in\{g>0\} \cap \widetilde{B}$, we get $\gamma \in \widetilde{B}_{W}$ and $g(\gamma)>0$, a contradiction, and similarly if $\tau \in\{g<0\} \cap \widetilde{A}$. Finally we apply Łojasiewicz's inequality [BCR, Lemma 7.7.10] to the functions $f$, $g$ and the set $\bar{T}$, so that there exists a nonnegative function $\varepsilon$ such that $F=f+\varepsilon g$ has the same sign as $f$ on $\bar{T}$ and the zero set of $\varepsilon$ is included in $\bar{T} \cap\{f=0\}$. In particular, by the remark just made we have that $\varepsilon$ does not vanish identically on $W$. Obviously $F$ separates $A$ and $B$ but it is unit in $\mathcal{R}(M)_{\mathcal{J}(W)}$ against our hypothesis on the odd character of $W$.

Coming back to our proof, we may thus assume that $\widetilde{A}_{W}$ and $\widetilde{B}_{W}$ are not separable. This means that there is an ordering $\gamma \in \Sigma_{W}$ with $\gamma \in\left(\widetilde{A}_{W}\right)^{\#} \cap$ $\left(\widetilde{B}_{W}\right)^{\#}=\widetilde{A}_{W}^{\#} \cap \widetilde{B}_{W}^{\#}$. Since $A$ and $B$ are separable, we have $\widetilde{A}^{\#} \cap \widetilde{B}^{\#}=\emptyset$ and taking the generalizations of $\gamma$ we get the two orderings $\sigma$ and $i \sigma$ of the statement, so that the proof is complete.

Finally we combine the above results to prove the main result of this section:

Theorem 3.8 (Universal obstruction to separation). $A$ and $B$ are not generically separable if and only if in some model (obtained from $M$ by a finite sequence of blowing-ups) there is a wall of $A$ and $B$ which is simultaneously odd and even.

Proof. If $A$ and $B$ are generically separable, it is immediate that there are no walls which are odd and even. So, assume that $A$ and $B$ are not generically separable. Then there exists a finite subspace of orderings $X \subset \Sigma_{K}$ such that $\widetilde{A} \cap X$ and $\widetilde{B} \cap X$ are not separable. Take $X$ with minimal cardinal. By Theorem 2.1 we may assume that $X$ is a GSO. Furthermore, by the minimality assumption we have that 


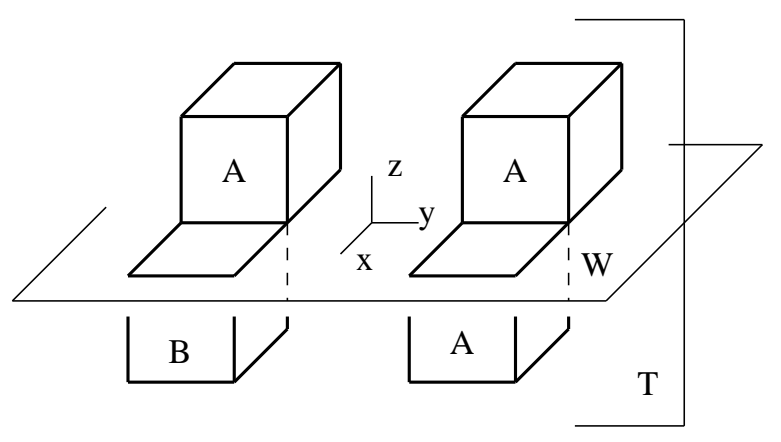

FiguRE 7

$X$ is an extension, say $X=Y\left[\mathbb{Z}_{2}\right]$, with $Y$ a geometric space of orderings. Now, take a model $N$ of $K$ in which $X$ is fully realized; cf. Proposition 1.4. Then $Y$ is a GSO of a hypersurface $W$ of $N$ which, as in the proof of Proposition 3.3, turns out to be a wall of $A$ and $B$. We claim that $W$ is an odd and even wall.

Indeed, again by the minimality assumption on $X$ we get that $X \subset \widetilde{A}^{\#} \cup \widetilde{B}^{\#}$. Moreover, since $\widetilde{A} \cap X$ and $\widetilde{B} \cap X$ are not separable there is $\sigma \in X$ with $\sigma \in$ $\widetilde{A}^{\#} \cap \widetilde{B}^{\#}$. Now $i \sigma \in X$, so that either $i \sigma \in \widetilde{A}^{\#}$ or $i \sigma \in \widetilde{B}^{\#}$. In any case, by the above proposition, we get that $W$ is odd and even.

Remark 3.9. a) Notice that Theorem 3.8 shows that any GSO of minimal cardinality in which $\widetilde{A}$ and $\widetilde{B}$ are not separated is centered at an odd and even wall in a model where it is fully realized.

b) From the above proof we derive the following simple fact which will be used later: if $A$ and $B$ are not generically separated, there is a fan $F=\{\alpha, i \alpha, \beta, i \beta\} \subset X$ such that, up to renaming, we have: $\alpha \in \widetilde{A}, \beta \in \widetilde{B}$ and $i \alpha, i \beta \in \widetilde{A}^{\#}$. Indeed, continuing with the argument at the end of the proof of the theorem, we have found $\sigma \in \widetilde{A}^{\#} \cap \widetilde{B}^{\#}$ with $i \sigma \in \widetilde{A}^{\#}$ or $i \sigma \in \widetilde{B}^{\#}$. Suppose the latter. Now, let $\beta \in \widetilde{B} \cap X$ and $\alpha \in \widetilde{A} \cap X$. From $i \beta=\sigma i \sigma \beta$ we get that $i \beta \in \widetilde{A}^{\#}$, and from $i \alpha=\sigma i \sigma \alpha$ we get that $i \alpha \in \widetilde{A}^{\#}$ which proves the claim.

c) The following example shows that the statement " $A$ and $B$ are not separable in $\Sigma_{W}\left[\mathbb{Z}_{2}\right]$ " is not equivalent to " $W$ is odd and even". Indeed, the sets $A$ and $B$ of Figure 7 are not separable (in fact they are not separable either in $\Sigma_{W}\left[\mathbb{Z}_{2}\right]$ or in $\Sigma_{T}\left[\mathbb{Z}_{2}\right]$, where $W$ and $T$ are the planes shown in the figure). However, neither $W$ nor $T$ is odd or even. An odd and even wall appears after blowing-up the line $T \cap W$.

d) Notice that if for a wall $W$ we have $\operatorname{Reg} W \cap(A \cup B) \neq \emptyset$, then $W$ is even (remember that $A$ and $B$ are open). Indeed if $a$ is a regular point of $W$ lying, say, in $A$, then a whole neighbourhood of $a$ is contained in $A$, which obviously implies that $W$ is even.

\section{THE GEOMETRIC CRITERION}

Despite the universal obstruction proved above, in order to decide the generic separation of two semialgebraic sets we want to fix a model of $M$ and develop a test in it, rather than considering all walls in all possible models of $K$. It turns out 
that the key property that we need for $A$ and $B$ (besides the nonsingularity of $M$ ) is that their walls are at normal crossings. Since we will work algebraically, by this we mean that if $f_{1}, \ldots, f_{s}$ is a family of polynomials describing $A$ and $B$, then all the $f_{i}$ 's are at normal crossings. We start by showing that under this condition we do not need to leave $M$ :

Theorem 4.1. Assume that $M$ is nonsingular and that all the walls of $A$ and $B$ are nonsingular and at normal crossings. Then $A$ are $B$ are generically separable if and only if $\widetilde{A}$ and $\widetilde{B}$ are separable in all subspaces $\Sigma_{W}\left[\mathbb{Z}_{2}\right]$ for all the walls $W$ of $A$ and $B$ in $M$.

Proof. Suppose that $A$ and $B$ are not generically separable. We may find a GSO, say $X$, of minimal cardinality in which $\widetilde{A}$ and $\widetilde{B}$ are not separable. Then by Proposition 1.4, Theorem 3.8 and Remark 3.9 a) there is a model $M^{\prime}$ of $K$, obtained blowing-up $M$, say $\pi: M^{\prime} \rightarrow M$, such that $X$ is centered at a wall $W^{\prime}$ of $A^{\prime}=$ $\pi^{-1}(A)$ and $B^{\prime}=\pi^{-1}(B)$ which is odd and even. This means that there is a 4-element fan $F=\{\sigma, i \sigma, \tau, i \tau\}$ in $\Sigma_{W^{\prime}}\left[\mathbb{Z}_{2}\right] \subset \Sigma_{K}$ with, say, $\sigma, i \sigma, i \tau \in \widetilde{A}^{\#}$ and $\tau \in \widetilde{B}$ and $\sigma \in \widetilde{A}$; cf. Remark 3.9 b). Set $R^{\prime}=\mathcal{R}\left(M^{\prime}\right), \mathfrak{q}=\mathcal{J}\left(W^{\prime}\right) R^{\prime}, R=\mathcal{R}(M)$, $T=\pi\left(W^{\prime}\right)$ and $\mathfrak{p}=\mathfrak{q} \cap R$, so that $\mathfrak{p}=\mathcal{J}(T) R$. Notice that $T$ lies in the Zariski boundary of $A \cup B$. We have that $R_{\mathfrak{q}}^{\prime}$ is a rank 1 discrete valuation ring with residue field $K\left(W^{\prime}\right)$ and $R_{\mathfrak{p}}$ is a regular local ring of dimension, say $d$. If $d=1$, then $\Sigma_{W^{\prime}}=\Sigma_{T}, T$ is again an odd and even wall and we are done. So, suppose that $d \geq 2$. Let $f_{1}, \ldots, f_{s}$ be a family of polynomials describing $A$ and $B$. Since the $f_{j}$ 's are normal crossings, there is a regular system of parameters $x_{1}, \ldots, x_{d}$ of the ring $R_{\mathfrak{p}}$ such that for all $j=1, \ldots, s$ we have

$$
f_{j}=a_{j} x_{1}^{m_{1}} \cdots x_{d}^{m_{d}}
$$

where $a_{j}$ is a unit in $R_{\mathfrak{p}}$, and we may assume that $\left\{x_{i}=0\right\}, i=1, \ldots, r, r \leq d$, correspond to the walls $W_{1}, \ldots, W_{r}$ of $A$ and $B$ passing through $T$.

Replacing $x_{i}$ by $-x_{i}$ we may assume that $x_{i}(\sigma)>0$ for all $i$. Now, let $t$ be a uniformizing parameter of the valuation ring $R_{\mathfrak{q}}^{\prime}$, so that, say, $t(\sigma)>0$ and $t(\tau)>0$. For each $i=1, \ldots, d$ we have $x_{i}=t^{r_{i}} v_{i}$ where $v_{i}$ is a unit in $R_{\mathfrak{q}}^{\prime}$.

We claim that some $f_{j}$ must change sign on $W^{\prime}$ (and in particular on some of the walls $\left.W_{1}, \ldots, W_{r}\right)$. Otherwise for any $\gamma \in \Sigma_{W^{\prime}}\left[\mathbb{Z}_{2}\right]$ all $f_{j}$ would have the same sign in $\gamma$ and $i \gamma$, and $\widetilde{A}$ and $\widetilde{B}$ would be saturated with respect to the specialization map $\Sigma_{W^{\prime}}\left[\mathbb{Z}_{2}\right] \rightarrow \Sigma_{W^{\prime}}$ which contradicts the minimality of $X$. In particular, for such a $j$, one of the exponents $m_{i} r_{i}$ must be odd for some $i \leq r$ and therefore both $m_{i}$ and $r_{i}$ are odd. Say $i=1$ and let us denote $W_{1}$ simply by $W$.

Now we distinguish two cases: assume first that the specializations $\bar{\sigma}$ and $\bar{\tau}$ of $\sigma$ and $\tau$ in $W^{\prime}$ are separated by some $a_{i}$, say $a_{1}$ (which in particular implies that they are already different in $T)$. Then, to any $\gamma \in(\widetilde{A} \cup \widetilde{B}) \cap \Sigma_{W^{\prime}}\left[\mathbb{Z}_{2}\right]$ we attach an ordering in $\Sigma_{W}\left[\mathbb{Z}_{2}\right]$ constructed as follows: $R_{\left(x_{1}\right)}$ is a discrete valuation ring with residue field the quotient field of $R /\left(x_{1}\right)$. In turn, $\left(R /\left(x_{1}\right)\right)_{\mathfrak{p}}=R_{\mathfrak{p}} /\left(x_{1}\right)$ is a regular local ring of dimension $d-1$ with $x_{2}, \ldots, x_{d}$ as parameters. Thus, we may consider the rank $d-1$ discrete valuation $v_{d-1}$ of the quotient field of $\left(R /\left(x_{1}\right)\right)$ dominating $R_{\mathfrak{p}} /\left(x_{1}\right)$ with value group $\Gamma=\bigoplus_{i=2}^{d} v_{d-1}\left(x_{i}\right) \mathbb{Z}\left[\mathrm{AnBrRz}\right.$, Lemma II.3.4]. Let $v_{d}$ be the composite of $R_{\left(x_{1}\right)}$ and $v_{d-1}$. In particular $v_{d}$ is a rank $d$ discrete valuation ring of $K$ with residue field $\kappa(\mathfrak{p})$, and for any ordering of $\Sigma_{\kappa(\mathfrak{p})}$ their pull-backs by $v_{d}$ are completely determined by the signs of $x_{1}, \ldots, x_{d}$ and belong to $\Sigma_{W}\left[\mathbb{Z}_{2}\right]$. 
Thus, given any ordering $\gamma \in \Sigma_{W^{\prime}}\left[\mathbb{Z}_{2}\right]$ we define $\widehat{\gamma}$ as the pull-back of $\bar{\gamma} \cap \kappa(\mathfrak{p})$ by $v_{d}$ determined by $x_{i}(\widehat{\gamma})=x_{i}(\gamma)$. Notice that the correspondence $\gamma \rightarrow \widehat{\gamma}$ is not in general bijective. However, for any $j=1, \ldots, s$ we have $f_{j}(\gamma)=f_{j}(\widehat{\gamma})$, so that if $\gamma$ was in $\widetilde{A}$ (resp. $\widetilde{B}$ ), so is $\widehat{\gamma}$. Now, an immediate checking shows that $\widehat{\sigma}, \widehat{i \sigma}, \widehat{\tau}, \widehat{i \tau}$ is a fan of $\Sigma_{W}\left[\mathbb{Z}_{2}\right]$ with $\widehat{\sigma} \in \widetilde{A}, \widehat{\tau} \in \widetilde{B}$ and $\widehat{\sigma}, \hat{i \sigma}, \widehat{i \tau} \in \widetilde{A}^{\#}$. This shows that $\widetilde{A}$ and $\widetilde{B}$ are not separable in $\Sigma_{W}\left[\mathbb{Z}_{2}\right]$ for the wall $W$, contrary to our assumption.

Second, suppose now that $\bar{\sigma}$ and $\bar{\tau}$ cannot be separated by any $a_{i}$ (for instance if $\bar{\sigma}=\bar{\tau}$ ). Then, since $\sigma$ and $\tau$ are separated by some $f_{j}$, there is another $x_{i}$, say $x_{2}$, such that $x_{2}(\tau)<0$. Thus, we have the following distribution of signs for $x_{1}$ and $x_{2}$ :

\begin{tabular}{c|cccc} 
& $\sigma$ & $i \sigma$ & $\tau$ & $i \tau$ \\
\hline$x_{1}$ & 1 & -1 & 1 & -1 \\
\hline$x_{2}$ & 1 & $\varepsilon$ & -1 & $-\varepsilon$ \\
\hline
\end{tabular}

where $\varepsilon=1$ or -1 . In any case we discover the shape of a four-element fan, and we proceed as above: for any $\gamma \in \Sigma_{W^{\prime}}\left[\mathbb{Z}_{2}\right]$ we define $\widehat{\gamma}$ as the pull-back of $\bar{\gamma} \cap \kappa(\mathfrak{p})$ by $v_{d}$ defined by $x_{i}(\widehat{\gamma})=x_{i}(\gamma)$. As before, an immediate checking shows that $\widehat{\sigma}, \widehat{i \sigma}, \widehat{\tau}, \widehat{i \tau}$ is a fan of $\Sigma_{W}\left[\mathbb{Z}_{2}\right]$ with $\widehat{\sigma} \in \widetilde{A}, \widehat{\tau} \in \widetilde{B}$ and $\widehat{\sigma}, \hat{i \sigma}, \hat{i \tau} \in \widetilde{A}^{\#}$. Therefore we get that $A$ and $B$ are not separable in $\Sigma_{W}\left[\mathbb{Z}_{2}\right]$ for the wall $W$, contrary to our assumption, and the proof is complete.

Next we develop a geometric criterion to test the separation of $A$ and $B$ in $\Sigma_{W}\left[\mathbb{Z}_{2}\right]$. We define the shadows of $A$ and $B$ in $W$ as the sets $A_{W}=\bar{A} \cap W$ and $B_{W}=\bar{B} \cap W$. We define the counter-shadows of $A$ and $B$ in $W$ as the shadows of the sets

$$
\begin{aligned}
& A^{t}=(A \cap\{t>0\}) \cup(B \cap\{t<0\}), \\
& B^{t}=(A \cap\{t<0\}) \cup(B \cap\{t>0\}),
\end{aligned}
$$

where $t$ is a uniformizer of the valuation $\operatorname{ring} \mathcal{R}(M)_{\mathcal{J}(W)}$. Notice that changing $t$ by $-t$ reverses the roles of $A^{t}$ and $B^{t}$. Thus, since we are working generically, up to reversing the roles of $A$ and $B$, the counter-shadows do not depend on the uniformizer.

The following immediate remark shows some useful relations:

Proposition 4.2. a) $f$ separates $A$ and $B$ generically if and only if $t f$ separates $A^{t}$ and $B^{t}$ generically.

b) $W$ is an even wall for $A$ and $B$ if and only if it is odd for $A^{t}$ and $B^{t}$.

If the shadows can be generically separated, then $\widetilde{A}$ and $\widetilde{B}$ can be separated in $\Sigma_{W}\left[\mathbb{Z}_{2}\right]$ and therefore $A$ and $B$ can be generically separated in a (infinitesimal) neighbourhood of $W$. However the converse is not true: take $A=\{z>0, y+1>0\}$ and $B=\{z<0,1-y>0\}$. Their shadows in $\{z=0\}$ cannot be separated, but still the function $z$ separates $A$ from $B$. Here is where the counter-shadows give a hand: they take care of this phenomena, and as the theorem below shows, shadows and counter-shadows together characterize the separation in $\Sigma_{W}\left[\mathbb{Z}_{2}\right]$.

In the context of geometric spaces of orderings $X=X^{\prime}\left[\mathbb{Z}_{2}\right]$, the shadow of a semialgebraic set $A$ corresponds to the set of specializations of $\widetilde{A}$ in $X^{\prime}$. Similarly, given two constructible subsets $\widetilde{A}, \widetilde{B} \subset X$, the counter-shadows are the 
specializations in $X^{\prime}$ of the sets:

$$
\begin{aligned}
& \widetilde{A}^{t}=\left(\widetilde{A} \cap\left(X^{\prime} \times\{1\}\right)\right) \cup\left(\widetilde{B} \cap\left(X^{\prime} \times\{i\}\right)\right), \\
& \widetilde{B}^{t}=\left(\widetilde{A} \cap\left(X^{\prime} \times\{i\}\right)\right) \cup\left(\widetilde{B} \cap\left(X^{\prime} \times\{1\}\right)\right) .
\end{aligned}
$$

We have the following general result:

Proposition 4.3. $\widetilde{A}$ and $\widetilde{B}$ are separable in $X=X^{\prime}\left[\mathbb{Z}_{2}\right]$ if and only if either their shadows or their counter-shadows are separable in $X^{\prime}$.

Proof. If the shadows are separable, say by a function $g^{\prime}$ on $X^{\prime}$, then $\left(1, g^{\prime}\right)$ separates $\widetilde{A}$ and $\widetilde{B}$. Analogously, if the counter-shadows are separable, then $\widetilde{A}^{t}$ and $\widetilde{B}^{t}$ are separable and therefore so are $\widetilde{A}$ and $\widetilde{B}$ by Proposition 4.2. Conversely, assume that $g$ separates $\widetilde{A}$ and $\widetilde{B}$ and denote by $\widetilde{A}^{\prime}$ and $\widetilde{B}^{\prime}$ respectively their specializations in $X^{\prime}$. If $g=\left(1, g^{\prime}\right)$, then $g^{\prime}$ separates $\widetilde{A}^{\prime}$ and $\widetilde{B}^{\prime}$. If $g=\left(i, g^{\prime}\right)$, then $i g=\left(1, g^{\prime}\right)$ separates $\widetilde{A}^{t}$ and $\widetilde{B}^{t}$ and therefore $g^{\prime}$ separates the counter-shadows of $\widetilde{A}$ and $\widetilde{B}$ in $X^{\prime}$.

As a consequence we get the following

Corollary 4.4. Let $A$ and $B$ be open semialgebraic. $\widetilde{A}$ and $\widetilde{B}$ are not separable in $\Sigma_{W}\left[\mathbb{Z}_{2}\right]$ if and only if neither the shadows nor their counter-shadows of $A$ and $B$ in $W$ are separable in $\Sigma_{W}$.

Winding up, taking into account Theorem 4.1 above we get the following geometric criterion for separation:

Theorem 4.5 (Separation criterion). Assume that $M$ is nonsingular and that the walls of $A$ and $B$ are at normal crossings. Then $A$ and $B$ are generically separable if and only if, for any wall $W$ of $A$ and $B$ in $M$, either the shadows or the countershadows of $A$ and $B$ are separable in $W$.

Proof. By Theorem $4.1 A$ and $B$ are separable if and only if they are separable in the spaces $\Sigma_{W}\left[\mathbb{Z}_{2}\right]$ for all walls $W$, and Corollary 4.4 states that this is equivalent to saying that either the shadows or the counter-shadows of $A$ and $B$ in $W$ are separable. So we are done.

Remark 4.6. a) The condition that the walls of $A$ and $B$ be at normal crossings is important. Notice that in the example of Figure 2 the shadows and countershadows of $A$ and $B$ are separated when they intersect only at the origin, while they are not after blowing-up.

b) The geometric criterion translates the problem of generic separation of $A$ and $B$ into a finite number of similar problems in one less dimension. This provides a recursive method to check generic separation, which in the end makes it a decidable problem by using Tarski's Principle as we will see in the next section.

\section{Decision Procedure FOR GENERIC SEPARATION}

We want to prove that the generic separation of two open semialgebraic subsets $A$ and $B$ of an algebraic variety $M \subset \mathbb{R}^{n}$ is decidable. Since we are concerned here with effective algorithms, we assume that $M$, as well as $A$ and $B$, are defined over $\mathbb{R}_{0}$, the field of real algebraic numbers. It is clear from Tarski's Transfer Principle [BCR, Proposition 5.2.3] that all the semialgebraic sets constructed in the sequel 
(like the irreducible components of the Zariski closure of the boundary of $A$ and $B$ ) can be defined as well over $\mathbb{R}_{0}$. Under this hypothesis we have:

Theorem 5.1. The generic separation of semialgebraic sets on an affine variety over the real numbers is decidable.

We will present two proofs. The first presents a tentative algorithm to test the separability, having $M, A$ and $B$ as input and answering YES or NO. It relies on the fact that the desingularization can be performed algorithmically [BiMi]. The rest of the operations needed (as computing closures of semialgebraic sets, computing the dimension of an algebraic set or finding its irreducible components) belong to the yoga of semialgebraic geometry and algebraic geometry algorithms; see [He], $[\mathrm{BeNe}],[\mathrm{Ne}]$. The second proof is more in the spirit of Model Theory and uses the notion of recursive enumerability. Both proofs work by induction on $d=\operatorname{dim} M$.

Notice that for $d=1, A$ and $B$ are generically separable (and separable since both notions coincide) if and only if $A \cap B=\emptyset$, which is a statement that can be expressed by a (formal) sentence in the first order language of ordered fields and therefore its truth in $\mathbb{R}$ is decidable by Tarski's result. Thus we assume that $\operatorname{dim} M=d>1$.

First proof.

Generic separation algorithm. Having $M, A$ and $B$ as input answers YES or NO to the question of whether they are generically separable:

1. Find a desingularization $\pi: M^{\prime} \rightarrow M$ and put $\overline{\partial A}^{Z} \cup \overline{\partial B}^{Z}$ into normal crossings. Set

$$
M \leftarrow M^{\prime}, \quad A \leftarrow \pi^{-1}(A), \quad B \leftarrow \pi^{-1}(B) .
$$

2. For all walls $W_{1}, \ldots, W_{r}$ of $A$ and $B$ check whether the shadows $A_{W_{i}}, B_{W_{i}}$ and the counter-shadows $A_{W_{i}}^{t}, B_{W_{i}}^{t}$ are generically separable (apply induction). If for some $i$ both are not generically separable, return "NO, $A$ and $B$ are not generically separable". Otherwise return "YES, they are generically separable".

Second proof. Again we work by induction on $\operatorname{dim} M$, so that we assume that decidability of generic separation is proved for varieties of dimension less than $d$.

For $\operatorname{dim} M=d$, it will be proved by recursively listing, on the one hand, all triples ( $M, A, B)$ where $M$ is a variety of dimension $d$ and $A$ and $B$ are open semialgebraic subsets of $M$ which can be generically separated in $M$, and on the other hand, the list of those triples $(M, A, B)$ where $A$ and $B$ cannot be generically separated in $M$.

Thus given a particular variety $M_{0}$ and a pair $A_{0}, B_{0}$ of open semialgebraic sets in $M_{0}$ the decision about (generic) separation is obtained by passing simultaneously through both lists. Depending on which side the triple $\left(M_{0}, A_{0}, B_{0}\right)$ will occur we know whether $A_{0}$ and $B_{0}$ are generically separable in $M_{0}$ or not.

The first list for generic separation is clearly contained in the list of quadruples $(M, A, B, p)$ of varieties $M$ of dimension $d$ and open semialgebraic sets $A$ and $B$ in $M$ which are generically separated by the polynomial $p$. This list of quadruples may be obtained in the following way. First, notice that by Bröcker's results (cf. $[\mathrm{Br} 4],[\mathrm{AnBrRz}$, Theorem V.2.17]), the number of polynomials needed to describe 
any semialgebraic subset of $M$ is bounded by a constant depending only on the dimension $d$ of $M$, so that we may recursively list all quadruples $(M, A, B, p)$ of all semialgebraic open sets $A$ and $B$ and all polynomials $p$. Then we use Tarski's decision procedure to eliminate those quadruples for which $p$ does not generically separate $A$ from $B$.

The second list will be contained in the following recursive list of certain finite sequences:

$$
\left(M, A, B, X,\left(\pi_{\nu}\right)_{\nu \leq m}, M^{\prime}, A^{\prime}, B^{\prime},\left(W_{j}^{\prime}, g_{j}\right)_{j \leq s}, X^{\prime}\right)
$$

where we have:

- $M \subset \mathbb{R}^{n}$ and $M^{\prime} \subset \mathbb{R}^{m}$ are compact varieties of dimension $d$,

- $X$ and $X^{\prime}$ are Zariski closed proper subsets of $M$ and $M^{\prime}$ respectively,

$-\pi=\left(\pi_{\nu}\right)_{\nu \leq n}$ is a biregular isomorphism of $M^{\prime} \backslash X^{\prime}$ and $M \backslash X$,

- $A$ and $B$ are open semialgebraic sets in $M$,

- $A^{\prime}$ and $B^{\prime}$ are open semialgebraic sets in $M^{\prime}$,

- $W_{1}^{\prime}, \ldots, W_{s}^{\prime}$ are the irreducible components of ${\overline{\partial A^{\prime}}}^{Z} \cup{\overline{\partial B^{\prime}}}^{Z}$,

$-g_{1}, \ldots, g_{s}$ are polynomials changing sign on $W_{1}^{\prime}, \ldots, W_{s}^{\prime}$ respectively.

Using the fact that it is decidable whether finitely many given algebraic sets form the components of the Zariski closure of a given semialgebraic set (cf. [He], $[\mathrm{BeNe}],[\mathrm{Ne}])$, it is clear that such a list can be recursively obtained.

Now we shorten this list so that eventually only the triples $(M, A, B)$ remain where $A$ and $B$ are not generically separable. We do this first by eliminating all sequences not satisfying one of the following conditions, which are, all of them, decidable.

We require that:

(i) $M^{\prime}$ is nonsingular,

(ii) $A^{\prime}=\operatorname{Int}\left(\overline{\pi^{-1}(A \backslash, X)}\right), B^{\prime}=\operatorname{Int}\left(\overline{\pi^{-1}(B \backslash X)}\right)$,

(iii) $W_{1}^{\prime}, \ldots, W_{s}^{\prime}$ are nonsingular and normal crossings.

Finally we save only those sequences which do not satisfy the separation criterion, that is, which satisfy the following condition which is decidable by the induction hypothesis, so that the procedure for generic separation is complete:

(a) for some $j \leq s$, the shadows of $A^{\prime}$ and $B^{\prime}$ as well as the shadows of $\left(A^{\prime}\right)^{g_{j}}$ and $\left(B^{\prime}\right)^{g_{j}}$ on $W_{i}^{\prime}$ are not generically separable.

We may ask whether given two generically separable semialgebraic sets $A$ and $B$, there is an upper bound on the degree of a separating polynomial in terms of their "complexity", that is, the total degree, size of the coefficients and number of polynomials of a description of $A$ and $B$. Assume that $\left\{B_{n}\right\}$ is a properly nested family, $B_{n} \subset B_{n+1}$, of open semialgebraic sets such that, for all $n, A$ and $B_{n}$ are separable while $A$ and $B=\bigcup_{n} B_{n}$ are not generically separable. Then the degrees of any sequence $\left\{f_{n}\right\}$ of polynomials separating $A$ and $B_{n}$ must go to infinity. Indeed, notice that scaling by the coefficient with larger absolute value we may always assume that the coefficients of $f_{n}$ lie in the unit ball. Then, if the degrees were bounded, say by $\rho \in \mathbb{N}$, we might represent each $f_{n}$ as a $\rho$-tuple in $[-1,1]^{\rho}$ and we would get a limit point $f$ which would represent a polynomial separating $A$ and $B$, a contradiction. 

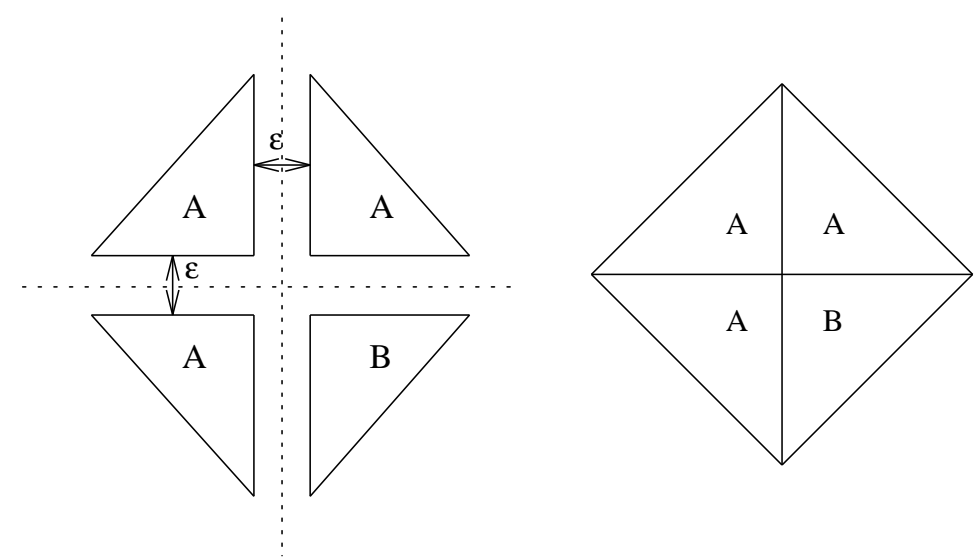

Figure 8

Also, by model completeness, it follows that there cannot exist a recursive bound for the degree of the separating function, since then the separation criterion 4.5 would be true for any real closed field $R$, while the following example shows that it fails when $R$ is nonarchimedean.

Example 5.2 (cf. [AnBrRz, example VI.7.10]). Let $R$ be any nonarchimedean real closed field, and consider the open semialgebraic subsets $A, B \subset R \times R$ as in the left-hand side of Figure 8, where $\varepsilon \in R$ is infinitesimal, i.e. $\varepsilon<\frac{1}{n}$ for all $n \in \mathbb{N}$. Notice that $A$ and $B$ satisfy the hypothesis of Theorem 4.5 , since $R \times R$ is nonsingular and the walls are nonsingular and normal crossings. However we claim that there is no polynomial separating $A$ and $B$, even generically.

For suppose that $h \in R[x, y]$ separates generically $A$ and $B$, and let $\mathcal{O}$ be the convex hull of $\mathbb{Q}$ in $R$. Then $\mathcal{O}$ is a convex valuation of $R$ whose maximal ideal $\mathfrak{m}$ is the set of infinitesimal elements. In particular $\varepsilon \in \mathfrak{m}$ and the residue field $R^{\prime}$ of $\mathcal{O}$ is an archimedean real closed field, so that it is a subfield of $\mathbb{R}$. Moreover, by scaling $h$ with the coefficient of larger absolute value in $R$ we may assume that $h \in \mathcal{O}[x, y]$ and that not all coefficients of $h$ lie in $\mathfrak{m}$. Now, the image $A^{\prime}, B^{\prime}$ of $A$ and $B$ in $R^{\prime}$ under the residue map looks like the right-hand side of Figure 8, and it is obvious that they are not generically separable in $R^{\prime}$. However, if $h^{\prime}$ is the image of $h$ in $R^{\prime}$ we have $h^{\prime} \neq 0$ and that it is, say $\geq 0$ on $A^{\prime}$ and $\leq 0$ on $B^{\prime}$, so that we get $h^{\prime} \in \mathfrak{m}[x, y]$, a contradiction.

Since besides Tarski's Principle the proof of the criterion depends on the density theorem of geometric spaces of orderings, we see that this approximation result holds only over $\mathbb{R}$. In fact, in the example above, there is a 4-element fan $F$ of $\Sigma_{R^{2}}$ in which the separation of $\widetilde{A}$ and $\widetilde{B}$ fails, which cannot be approximated by any geometrical one.

\section{Generic separation versus separation}

In this section $A$ and $B$ are arbitrary semialgebraic subsets of an algebraic variety $M$. We recall that the boundary $\partial A$ of $A$ is defined as $\partial A=\bar{A} \backslash \operatorname{Int}(A)$. In particular, all points where $\operatorname{dim} A<\operatorname{dim} M$ belong to $\partial A$. 


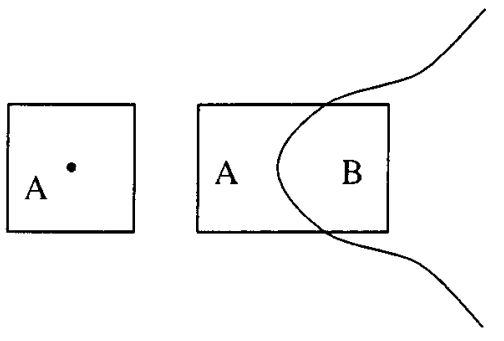

FiguRE 9

Obviously, separation implies generic separation, but the converse is not true as is easily seen by taking for instance the sets in $\mathbb{R}^{2}$ :

$$
\begin{aligned}
& A=\{(x, y) \mid 0<x<1,0<y<1\} \cup\left\{(x, y) \mid 0<x<\frac{1}{2}, y=0\right\}, \\
& B=\{(x, y) \mid 0<x<1,-1<y<0\} \cup\left\{(x, y) \mid \frac{1}{2}<x<1, y=0\right\}
\end{aligned}
$$

or the sets $A, B \subset \mathbb{R}^{2}$ of Figure 9, where the curve is a cubic with an isolated point.

In both cases the idea is the same: any function separating generically $A$ and $B$ must vanish at certain points lying in $A \cup B$ and therefore they cannot be separated in the sense of Definition 3.1. Thus we want to identify the set of common zeros of the family of functions which separate generically $A$ and $B$.

To start with, notice that any function $f$ which separates $A$ and $B$ generically must vanish identically on $\bar{A} \cap \bar{B}$ and therefore on ${\overline{\bar{A} \cap \bar{B}^{2}}}^{Z}$. In fact, this is only a part of the set of points in which any function separating $A$ and $B$ must vanish. For, let $W$ be any irreducible algebraic subset of $M$, such that the shadows of $A$ and $B$ in $W$ are not generically separable. Then any function $f$ which separates $A$ and $B$ must vanish identically on $W$ since it must be $\geq 0$ on $A_{W}$ and $\leq 0$ on $B_{W}$. We denote by $Z$ the union of all these sets and we name it the separation nullspace of $A$ and $B$. Notice that in particular we have $\overline{\bar{A}} \cap \bar{B}^{Z} \subset Z$ and that a necessary condition for $A$ and $B$ to be separable is that $Z \cap(A \cup B)=\emptyset$.

The following result extends Theorem 3.3 of $[\mathrm{AcBgFo}]$ and shows that separation and generic separation coincide up to the separation nullspace $Z$. Compare also with $[\mathrm{AnBrRz}$, Theorem V.3.2 $a), b)]$.

Theorem 6.1. With the notations above, assume that $Z \cap(A \cup B)=\emptyset$. Then $A$ and $B$ are separable if and only if they are generically separable.

Proof. One direction is trivial. For the other, we assume that there exists $f$ which separates generically $A$ and $B$ in $M$, that is, there is a proper algebraic set $W$ such that $\left.f\right|_{A \backslash W}>0$ and $\left.f\right|_{B \backslash W}<0$. We will see that we can find $f^{\prime}$ which separates $A$ and $B$ up to a proper algebraic subset $W^{\prime} \subset W$. Thus the proof ends by induction.

First of all notice that from our assumption on $Z$ we may assume that no irreducible component of $W$ lies in $Z$. Also, replacing $W$ by $\overline{\{f=0\} \cap(A \cup B)}^{Z}$, we may assume that $W=\overline{W \cap(A \cup B)}^{Z}$ and that $f$ vanishes on $W$. Now, take any irreducible component of $W$ of maximum dimension, which we still denote by $W$. Since $W$ is not in $Z$, there is a function $g$ which separates generically the shadows $A_{W}$ and $B_{W}$, say $\left.g\right|_{\left(A_{W} \backslash W^{\prime}\right)}>0$ and $\left.g\right|_{\left(B_{W} \backslash W^{\prime}\right)}<0$. Consider the semialgebraic 


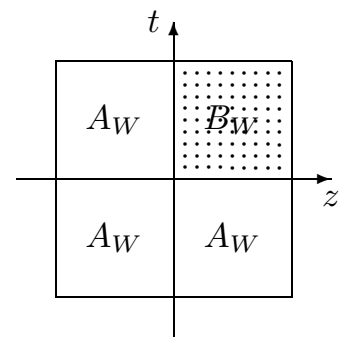

FiguRE 10

set $S=(\bar{A} \backslash\{g>0\}) \cup(\bar{B} \backslash\{g<0\})$. By Lojasiewicz's inequality [BCR, Lemma 7.7.10] there is a nonnegative function $\varepsilon$ such that $f^{\prime}=f+\varepsilon g$ has the same sign as $f$ over $S$, and with the zero set of $\varepsilon$ contained in $\overline{\{f=0\} \cap S}^{Z}$. Thus we get that $\left.f^{\prime}\right|_{A \backslash W^{\prime}}>0$ and $\left.f^{\prime}\right|_{B \backslash W^{\prime}}<0$ and we are done.

Remark 6.2. a) If $\operatorname{dim} M=2$, then $Z={\overline{\bar{A} \cap \bar{B}^{2}}}^{Z}$. If $\operatorname{dim} M=3$ and $M$ is nonsingular, in [AcBgFo] it is shown that $Z$ is the union of ${\overline{\bar{A} \cap \bar{B}^{2}}}^{Z}$ and the odd walls of $A$ and $B$. However in higher dimensions this is no longer true as the following example shows: Let

$$
\begin{aligned}
A= & \{-1<x<0,-1<y<0,-1<z<1,-1<t<0\} \cup \\
& \{-1<x<0,-1<y<0,-1<z<0,0<t<1\}, \\
B= & \{0<x<1,0<y<1,0<z<1,0<t<1\} .
\end{aligned}
$$

Notice that the shadows of $A$ and $B$ in $W=\{x=y=0\}$ are the sets of Figure 10 , which are not generically separable. Thus, $Z$ contains $W$, although none of the walls of $A$ and $B$ are odd, as we will see later.

b) Theorem 6.1 shows that if we consider the sets $A^{*}=\bar{A} \cup Z$ and $B^{*}=\bar{B} \cup Z$, then $A$ and $B$ are separable if and only if $A^{*}$ and $B^{*}$ are separable in the sense of [AnBrRz, Definition V.3.1].

c) Also Theorem 6.1 shows that $Z$ coincides with the set of common zeros of all functions separating generically $A$ and $B$. This is the reason why we call $Z$ the separation nullspace of $A$ and $B$ in $M$.

The next result shows that $Z$ is contained in the union of $\operatorname{Sing} M$ and the Zariski closure of the boundary of $A \cup B$.

Theorem 6.3. Let $A$ and $B$ be two semialgebraic subsets of $M$ such that

$$
\left(\overline{\partial A}^{Z} \cup \overline{\partial B}^{Z} \cup \operatorname{Sing} M\right) \cap(A \cup B)=\emptyset .
$$

Then $A$ and $B$ are separable if and only if they are generically separable. In particular $Z \subset \overline{\partial A}^{Z} \cup \overline{\partial B}^{Z} \cup \operatorname{Sing} M$.

Proof. Suppose that $A$ and $B$ are not separable but are generically separable. Then, by Theorem 6.1, there is a subvariety $T \subset Z$ such that $(A \cup B) \cap T \neq \emptyset$ and the shadows $A_{T}$ and $B_{T}$ of $A$ and $B$ in $T$ are not generically separable. By Brocker's theorem, this means that there is a finite subspace of orderings $Y \subset \Sigma_{T}$ such that $\widetilde{\left(A_{T}\right)} \cap Y$ and $\widetilde{\left(B_{T}\right)} \cap Y$ are not separable. In particular we have $A_{T} \neq \emptyset$ and $B_{T} \neq \emptyset$. Now, by our hypothesis we get $T \not \subset \overline{\partial A}^{Z} \cup \overline{\partial B}^{Z}$ and therefore $A_{T} \subset A$ 
and $B_{T} \subset B$. Let $\mathfrak{p}$ be the ideal of functions vanishing on $T$. Since $T \not \subset \operatorname{Sing} M$, the localization $\mathcal{R}(M)_{\mathfrak{p}}$ is a regular local ring with residue field $\mathcal{K}(T)$. Thus, there is a discrete valuation ring $V$ of $K$ dominating $\mathcal{R}(M)_{\mathfrak{p}}$ and with residue field $K(T)$. Fix any sign for the regular system of parameters of $\mathcal{R}(M)_{\mathfrak{p}}$ and consider the pull-back of $Y$ to $K$ with these parameters fixed. We get a subspace of orderings $X \subset \Sigma_{K}$ isomorphic to $Y$. Moreover, if $\sigma \in X$ is the unique generalization of $\tau \in Y$, we have that $\sigma \in \widetilde{A}$ (resp. $\sigma \in \widetilde{B}$ ) if and only if $\tau \in \widetilde{\left(A_{T}\right)} \cap Y$ (resp. $\tau \in \widetilde{\left(B_{T}\right)} \cap Y$ ) and we get that $\widetilde{A}$ and $\widetilde{B}$ are not separable in $X$, and therefore neither are they in $\Sigma_{K}$, in contradiction with our assumption that $A$ and $B$ were generically separable.

As an application we see that in the normal crossing situation of the separation criterion 4.5 the notions of separation and generic separation coincide for open sets.

Corollary 6.4. Assume that $A$ and $B$ are open, $M$ is nonsingular and all the walls are nonsingular and normal crossings. Then $A$ are $B$ are separable if and only if they are generically separable.

Proof. Assume that $A$ and $B$ are generically separable. We will see that then $Z \cap(A \cup B)=\emptyset$, which by Theorem 6.1 implies that $A$ and $B$ are separable. We work by induction on $\operatorname{dim} M$. If $\operatorname{dim} M=1$, the claim is trivial. So assume that $d=\operatorname{dim} M>1$ and that $Z \cap(A \cup B) \neq \emptyset$. Let $T \subset Z$ be an irreducible algebraic set with $T \cap(A \cup B) \neq \emptyset$. Since $M$ is nonsingular, by Theorem 6.3 we have that $T \subset \overline{\partial A}^{Z} \cup \overline{\partial B}^{Z}$, so that $T$ is contained in a wall, say $W$. In particular $W \cap(A \cup B) \neq \emptyset$ and since $W$ is nonsingular, by Remark $3.9 \mathrm{~d})$, we have that $W$ is even. Thus, for any function $f$ separating generically $A$ and $B$, in $\mathcal{R}(M)_{\mathcal{J}(W)}$ we have $f=t^{m} u$ where $t$ is a uniformizer, $u$ a unit and $m$ is even. Hence $u$ still separates $A$ and $B$ generically and being a unit, it separates also $A_{W}$ and $B_{W}$.

Since $W$ is nonsingular and the walls of $A_{W}$ and $B_{W}$ are also nonsingular and normal crossings, we may apply the induction hypothesis to $W, A_{W}$ and $B_{W}$. In particular, since $A_{T}=\left(A_{W}\right)_{T}$ and $B_{T}=\left(B_{W}\right)_{T}$ we have that $T$ is also in the separation nullspace of $A_{W}$ and $B_{W}$ and by induction we get that $T \cap\left(A_{W} \cup B_{W}\right)=$ $\emptyset$. But $T \cap(A \cup B) \subset T \cap\left(A_{W} \cup B_{W}\right)$ and we get a contradiction.

The following example shows that the result of Theorem 6.3 cannot be improved.

Example 6.5. Let $Q$ be an irreducible compact curve in $\mathbb{R}^{2}$ with two connected components $Q_{1}$ and $Q_{2}$ which are separated by the line $x_{1}=0$. Then consider the cone $M \subset \mathbb{R}^{5}$ constructed over $Q$ placed at the plane $x_{3}=x_{4}=x_{5}=1$ and taking as vertex the 2 -plane $M_{0}$ with coordinates $x_{4}, x_{5}$. Thus, $M=M_{1} \cup M_{2}$ where $M_{i}$ is the cone over $Q_{i}$, and the polynomial $x_{1} x_{5}$ separates $M_{1}$ and $M_{2}$ outside the vertex $M_{0}$, so that they are generically separated. On $M_{0}$ we consider the semialgebraic sets

$$
\begin{aligned}
& A_{0}=\left\{-1<x_{4}<0,-1<x_{5}<1\right\} \cup\left\{0<x_{5}<1,-1<x_{4}<1\right\} \\
& B_{0}=\left\{0<x_{4}<1,-1<x_{5}<0\right\} .
\end{aligned}
$$

Next, we define $A$ as the union of all the generatrices of the cone connecting $Q_{1}$ and $A_{0}$, and $B$ as the union all the generatrices of the cone connecting $Q_{2}$ and $B_{0}$. Then $A$ and $B$ are open semialgebraic sets in $M$, generically separated by $x_{1} x_{5}$ but $A \cap M_{0}$ and $B \cap M_{0}$ are not. So, $M_{0}=\operatorname{Sing} M=Z$.

Although Theorem 6.3 does not identify the separation nullspace, once we know that $A$ and $B$ are generically separated, it opens the door to decide whether $Z \cap$ 
$(A \cup B)=\emptyset$. Indeed, let $T \subset Z$ be an irreducible subvariety with $T \cap(A \cup B) \neq \emptyset$ and maximal dimension. Then $T$ is contained in some irreducible component $W$ of $\left(\overline{\partial A}^{Z} \cup \overline{\partial B}^{Z} \cup \operatorname{Sing} M\right)$. In particular $W \cap(A \cup B) \neq \emptyset$. Now, if the shadows $A_{W}$, $B_{W}$ are not generically separated, then $W$ itself is in $Z, W=T$ and we are done with the test. So assume that $A_{W}$ and $B_{W}$ are generically separated. Notice that $\left(A_{W}\right)_{T}=A_{T},\left(B_{W}\right)_{T}=B_{T}$ and $T \cap(A \cup B) \subset T \cap\left(A_{W} \cup B_{W}\right)$ since $A \cap W \subset A_{W}$ and $B \cap W \subset B_{W}$. Thus in particular $T$ is contained in the separation nullspace of $A_{W}$ and $B_{W}$ in $W$ which by Theorem 6.3 is contained in $\left({\overline{\partial A_{W}}}^{z} \cup{\overline{\partial B_{W}}}^{z} \cup \operatorname{Sing} W\right)$. Hence $T$ must be contained in some irreducible component of this set, which has codimension at least 1 in $W$. Iterating this process, we get that $T$ must be one of the irreducible varieties appearing in the finite list $\mathcal{L}_{M, A, B}$ obtained in this way, which we systematize in the following algorithm:

Producing the list $\mathcal{L}_{M, A, B}$ of obstructions to separation.

1. Initialize $\mathcal{L}_{M, A, B} \leftarrow\{\emptyset\}, M_{0} \leftarrow M, A_{0} \leftarrow A$ and $B_{0} \leftarrow B$.

2. Add to $\mathcal{L}_{M, A, B}$ all the irreducible components $W$ of $\operatorname{Sing}\left(M_{0}\right) \cup\left({\overline{\partial A_{0}}}^{z} \cup{\overline{\partial B_{0}}}^{z}\right)$ which intersect $A \cup B$.

3. For the new $W \in \mathcal{L}_{M, A, B}$ IF $\operatorname{dim} W>1$, set $M_{0} \leftarrow W, A_{0} \leftarrow A_{W}$ and $B_{0} \leftarrow B_{W}$ and GOTO 2 .

Finally, bringing together the generic separation decision procedure and the procedure for the list $\mathcal{L}_{M, A, B}$ we can design a procedure to decide the separation of $A$ and $B$, proving the desired result:

Theorem 6.6. The separation of semialgebraic sets on an affine variety over the real numbers is decidable.

Separation Decision Procedure. Having $M, A$ and $B$ as input answers YES or NO to the question of whether they are separable:

1. IF $A$ and $B$ are not generically separable, return "NO, $A$ and $B$ are not separable".

2. IF for some $W \in \mathcal{L}_{M, A, B}$ the shadows $A_{W}$ and $B_{W}$ are not generically separable, return "NO, $A$ and $B$ are not separable".

3. ELSE return "YES, $A$ and $B$ are separable".

\section{REFERENCES}

[AcAnBg] F. Acquistapace, C. Andradas, F. Broglia: "Classification of obstructions for separation of Semialgebraic Sets in dimension 3", Rev. Matematica U.C.M. 10 (número suplementario) (1997) 27-49. CMP 98:05

[AcBgFo] F. Acquistapace, F. Broglia, E. Fortuna: "A separation theorem in dimension 3", Nagoya Math. Journal 143 (1996) 171-193. MR 97k:14056

[AnBrRz] C. Andradas, L. Bröcker, J.M. Ruiz: Constructible sets in real geometry, Ergeb. Math. 33, Springer-Verlag Berlin-Heidelberg-New York, 1996. MR 98e:14056

[AnRz1] C. Andradas, J.M. Ruiz: "More on basic semialgebraic sets", in Real Algebraic and analytic geometry, Lect. Notes in Math. 1524, Springer-Verlag, New York, (1992), 128-139. MR 94g: 14030

[AnRz2] C. Andradas, J.M. Ruiz: "Low dimensional sections of basic semialgebraic sets", Illinois J. of Math. 38 (1994), 303-326. MR 95d:14056

[AnRz3] C. Andradas, J.M. Ruiz: "Ubiquity of Łojasiewicz example of a non-basic semialgebraic set", Michigan Math. J. 41 (1994). MR 96e:14064

[BeNe] E. Becker, R. Neuhaus: "Computation of real radicals of polynomial ideals", Proc. MEGA 92, Nice, France, Birkhauser (1993) 1-20. MR 94g:12001 
[BiMi] E. Bierstone, P. Milman: "Canonical desingularization in characteristic zero by blowing-up the maximum strata of a local invariant", Inventiones Math. 128 (1997), 207-302. MR 98e: 14010

[BCR] J. Bochnak, M. Coste, M.-F. Roy: Géométrie algébrique réelle, Ergeb. Math. 12, Springer-Verlag, Berlin-Heidelberg-New York, 1987. MR 90b:14030

[BoEf] J. Bochnak, G. Efroymson: "Real Algebraic geometry and the Hilbert 17th problem". Math. Ann. 251 (1980) 213-241. MR 81k:14023

[Br1] L. Bröcker: "Spaces of Orderings and semialgebraic sets", Canadian Math. Society conference proc. 4 (1984) 231-248. MR 86m:12002

[Br2] L. Bröcker: "Characterization of fans and hereditarily pythagorean fields", Math. Z. 151 (1976) 149-163. MR 54:10224

[Br3] L. Bröcker: "On the separation of basic semialgebraic sets by polynomials" Manuscripta Math. 60 (1988) 497-508 MR 89d:14034

[Br4] L. Bröcker: "On basic semialgebraic sets", Expo. Math. 9 (1991) 289-334. MR 93b: 14085

[BrSt] L. Bröcker, G. Stengle: "On the Mostowski number", Math. Z. 203 (1990) 629-633. MR 91g:14058

[He] G. Hermann: "Die Frage der endlich vielen Schritte in der Theorie der Polynomideale", Math. Annalen 95 (1926).

[Hk] H. Hironaka: "Resolution of singularities of an algebraic variety over a field of characteristic zero", Annals of Math. 79 (1964) I:109-123, II:205-326. MR 33:7333

[Mo] T. Mostowski: "Some properties of the ring of Nash functions", Ann. Scuola Norm. Sup. Pisa 3 (1976) 245-266. MR 54:307

[Mr1] M. Marshall: "Classification of finite spaces of orderings", Canad. J. Math. 31 (1979) 320-330. MR 80i:10026

[Mr2] M. Marshall: "Quotients and inverse limits of spaces of orderings", Canad. J. Math. 31 (1979) 604-616. MR 80f:10021

[Mr3] M. Marshall: "The Witt ring of a space of orderings", Trans. Amer. Math. Soc. 258 (1980) 505-521. MR 81b:10012

[Mr4] M. Marshall: "Spaces of orderings IV", Canad. J. Math. 32 (1980) 603-627. MR 81m:10035

[Mr5] M. Marshall: "Spaces of orderings and Abstract Real Spectra", Lect. Notes in Math. 1636, Springer-Verlag, New York, (1997). MR 98b:14041

[Ne] R. Neuhaus: "Computation of real radicals of polynomial ideals II", Proc. MEGA 92, Nice, France, Birkhauser (1993). MR 94g:12001

[Pr] A. Prestel: "Model Theory for the Real Algebraic Geometer", to appear as a Quaderni del Dottorato del Dipartimento de Matematica, Università di Pisa (1998).

[Rz] J. Ruiz: "A note on a separation problem", Archiv der Mathematik 43 (1984) 422-426. MR 86f:32007

Dipartimento di Matematica, Università di Pisa, Via Buonarroti 2, 56127 Pisa, Italy

E-mail address: acquistf@gauss.dm.unipi.it

Departamento de Algebra, Facultad de Matemáticas, Universidad Complutense, 28040 MADRID, SPAIN

E-mail address: andradas@sunal1.mat.ucm.es

Dipartimento di Matematica, Università di Pisa, Via Buonarroti 2, 56127 Pisa, italy

E-mail address: broglia@gauss.dm.unipi.it 